García Cruz, J.I.; Febles Ramírez, M.F.; Pestana Pérez, G. (2017): “Las Tecnologías de la Información Geográfica aplicadas a la sistematización de los sistemas agrarios. Bases para el estudio de su dimensión espacial y temporal. El caso del mapa de cultivos de canarias (España)", GeoFocus (Artículos), no 19, p. 181-206. ISSN: 1578-5157 http://dx.doi.org/10.21138/GF.558

\title{
LAS TECNOLOGÍAS DE LA INFORMACIÓN GEOGRÁFICA APLICADAS A LA SISTEMATIZACIÓN DE LOS SISTEMAS AGRARIOS. BASES PARA EL ESTUDIO DE SU DIMENSIÓN ESPACIAL Y TEMPORAL. EL CASO DEL MAPA DE CULTIVOS DE CANARIAS (ESPAÑA)
}

\author{
JUAN ISRAEL GARCÍA CRUZ ${ }^{1}$, MIGUEL FRANCISCO FEBLES RAMÍREZ², GUSTAVO \\ PESTANA PÉREZ ${ }^{3}$ \\ ${ }^{1}$ Escuela Universitaria de Turismo de Santa Cruz de Tenerife (Adscrita a la Universidad de La \\ Laguna). Calle José Zárate y Penichet no 5, 38001 Santa Cruz de Tenerife (España) \\ jgarciac@ull.edu.es \\ ${ }^{2}$ GEODOS. Planificación y Servicio S.L. Calle Emilio Calzadilla, 5, 3 C, 38002, Santa Cruz de \\ Tenerife, España. \\ miguelfebles@geodos.es \\ ${ }^{3}$ Servicio Técnico de Planificación de Obras y Ordenación Rural. Consejería de Agricultura, \\ Ganadería, Pesca y Aguas. Gobierno de Canarias. \\ gpesper@gobiernodecanarias.org
}

\section{RESUMEN}

Los sistemas agrarios sintetizan un fenómeno territorial complejo, heterogéneo y dinámico que precisa de sistemas de información adaptados a estas circunstancias para su comprensión y gestión. En el caso de Canarias, la singularidad de sus entornos insulares ha demandado el desarrollo de un sistema propio, diseñado e implementado con el apoyo de Tecnologías de la Información Geográfica (TIG), dando como resultado el Mapa de Cultivos de Canarias.

El presente trabajo, considerando las particularidades del contexto canario, resume los antecedentes que han influido en su desarrollo, implementado a través de un Sistema de Información Geográfica (SIG), culminando con su viabilidad en el tratamiento de la dimensión espacial y temporal de los sistemas agrarios insulares. Así, se evidencia que su aplicabilidad va más allá de las necesidades iniciales de la administración que la gestiona, pudiéndose extender a la ordenación del territorio o el estudio del paisaje, entre otros campos, dado que el uso combinado de las dimensiones espacial y temporal enriquece la lectura que puede realizarse del territorio.

Palabras clave: Sistema agrario, mapa de cultivos, sistema de información geográfica, dimensión espacial, dimensión temporal. 
García Cruz, J.I.; Febles Ramírez, M.F.; Pestana Pérez, G. (2017): "Las Tecnologías de la Información Geográfica aplicadas a la sistematización de los sistemas agrarios. Bases para el estudio de su dimensión espacial y temporal. El caso del mapa de cultivos de canarias (España)", GeoFocus (Artículos), $n^{\circ}$ 19, p. 181-206. ISSN: 1578-5157 http://dx.doi.org/10.21138/GF.558

GEOGRAPHIC INFORMATION TECHNOLOGIES APPLIED TO THE SYSTEMATIZATION OF THE FARMING SYSTEM. BASES FOR STUDYING THEIR SPATIAL AND TEMPORAL DIMENSIÓN. THE CASE OF THE CROP MAP OF CANARY ISLANDS (SPAIN)

\section{ABSTRACT}

The farming system synthesize a complex, heterogeneous and dynamic territorial phenomenon that requires information systems adapted to these circumstances for their understanding and management. In the case of the Canary Islands, the singularity of their insular contexts has demanded the development of an own system, designed and implemented with the support of Geographic Information Technologies (GIT), resulting in the Map of Crops of the Canary Islands.

The present paper, considering the particularities of the Canary context, summarizes the antecedents that have influenced this development, implemented through a Geographic Information System (GIS), culminating with viability in the treatment of the spatial and time dimension of the island's farming systems. Thus, it is evident that its applicability goes beyond the specific needs of the administration that manages it, being able to extend to the planning of the territory or the study of the landscape, among other fields, since the combined use of the space and time dimensions enriches the reading that can be made of the territory.

Keywords: Farming system, crop map, geographical information system, spatial dimension, time dimension.

\section{Introducción. La complejidad de los sistemas agrarios y la necesidad de fuentes para su entendimiento, análisis y gestión}

Un sistema agrario, agrosistema o sistema de producción agraria puede definirse como el conjunto de técnicas empleadas para la producción de alimentos, mediante la utilización del suelo o tecnologías que lo sustituyan (como en el caso de los hidropónicos), considerando las condiciones bioclimáticas estacionales o moderando su efecto mediante técnicas que las moderan. Ésta se lleva a cabo mediante una combinación de cultivos o cultivos y ganadería, más o menos diversificados, que hacen uso de una serie de técnicas de cultivo (rotación, regadío, ecológica, intensiva, extensiva, etc.), medios técnicos de producción (abonos, productos fitosanitarios, maquinaria, etc.), y medios humanos (mano de obra) para su desarrollo ${ }^{1}$. De esta forma, el concepto se asocia a la forma de hacer agricultura, lo que supone la consideración de su dimensión económica, técnica, sociocultural y medioambiental a la hora de caracterizar y diferenciar cada uno de ellos (Parra, et.al., 2004). Desde este punto de vista, se asocia a una unidad geográfica que combina una serie de hechos naturales, históricos, sociales, económicos, jurídicos y técnicos que influyen en los métodos de cultivo y el paisaje (expresión física) que puede dar lugar (Egea, 2005).

En el sistema se deben tener en cuenta tanto los objetos como sus relaciones. Así, los objetos pueden ser caracterizados individualmente mediante su localización, forma y atributos, mientras que, las relaciones permiten definir el sistema que los vincula entre sí. En este sentido, los objetos dependen y se relacionan entre ellos, dando lugar a algo más que la mera suma de las partes.

\footnotetext{
${ }^{1}$ Adaptado de Lacoste (1983) en Aguilera, et. al. (2012).
} 
García Cruz, J.I.; Febles Ramírez, M.F.; Pestana Pérez, G. (2017): "Las Tecnologías de la Información Geográfica aplicadas a la sistematización de los sistemas agrarios. Bases para el estudio de su dimensión espacial y temporal. El caso del mapa de cultivos de canarias (España)", GeoFocus (Artículos), no 19, p. 181-206. ISSN: 1578-5157 http://dx.doi.org/10.21138/GF.558

Esto supone la identificación de conjuntos con lógica interna, una organización que responde a un objetivo común, lo que lo convierte en un todo, esto es, un sistema agrario (Egea, 2005).

Partiendo de este planteamiento, cada contexto socio-económico y territorial puede definir un escenario que condiciona directa o indirectamente al sistema agrario desarrollado en él, forzando su evolución en la medida que éste experimenta cambios. De este modo, el conjunto de cultivos que se desarrolla en un determinado territorio es el resultado de una lógica más amplia que la mera unidad de explotación, en la que los tipos de cultivo, su periodicidad, rotación, distribución, etc., responderán al funcionamiento interno de un sistema agrario que los engloba, a la vez que los diferencia de otros conjuntos o sistemas. Por esta razón, el entendimiento del sistema, su análisis, así como la articulación de una gestión activa de estos espacios, debe partir de un conocimiento lo más exhaustivo posible de los mismos.

Ante la necesidad de entender un sistema agrario, medir y caracterizar los objetos y sus relaciones son labores clave. En este sentido, diversas fuentes a la lo largo de la historia han tratado de aportar conocimientos, en función de las condiciones técnicas, conceptuales y tecnológicas de cada momento, lo que ha llevado a su continua evolución. Así, largo es el recorrido desde las primeras estadísticas agropecuarias de finales del siglo XVIII, la consideración de la representación cartográfica a partir de finales del siglo XIX, hasta la reciente incorporación de las Tecnologías de la Información Geográfica (TIG) entre finales del siglo XX y principios del XXI, cuestión que trataremos a continuación de forma resumida.

\section{Antecedentes. La evolución de las fuentes destinadas a su sistematización y entendimiento}

A la hora de plantear el estudio o la gestión de estos sistemas, son las administraciones públicas quienes han jugado un papel predominante o protagonista en la proposición e implementación de fuentes destinadas a este fin. Así, tradicionalmente se ha optado por el desarrollo de aproximaciones estadísticas, a las que posteriormente se les uniría la representación cartográfica, incrementando progresivamente el peso de la visión espacial del fenómeno.

Las primeras referencias identificadas se remiten a los trabajos estadísticos realizados en Gran Bretaña a mediados del siglo XVIII, teniendo como referencia los de Palluel en 1788 (Alonso, 1990), lo que llevaría a la posterior implantación de organismos públicos dedicados al seguimiento del sector agropecuario a lo largo del siglo XIX.

En el ámbito estatal, el primer inventario de riqueza agrícola se llevaría a cabo en 1748, a través de un proyecto del Marqués de la Ensenada, continuando posteriormente con la creación de la Oficina de Estadísticas en 1802 y la Comisión de Estadística General del Reino en 1856; transformada más adelante en la Junta General de Estadística (1861), a la que se le vinculaba una dirección general dedicada a la riqueza territorial y pecuaria. En 1887 se hacía público el Reglamento Orgánico del Cuerpo de Ingenieros Agrónomos, a los que se les asigna la responsabilidad de realizar las estadísticas agrícolas y pecuarias. A partir de este momento comienza a desarrollarse reglas que ordenan la recogida de datos y la elaboración de formatos para su difusión, lo que daría lugar a la aparición, a mediados de siglo, de publicaciones periódicas, como los Anuarios Estadísticos, en los que se reservaba un espacio al sector agrario. 
García Cruz, J.I.; Febles Ramírez, M.F.; Pestana Pérez, G. (2017): “Las Tecnologías de la Información Geográfica aplicadas a la sistematización de los sistemas agrarios. Bases para el estudio de su dimensión espacial y temporal. El caso del mapa de cultivos de canarias (España)", GeoFocus (Artículos), no 19, p. 181-206. ISSN: 1578-5157 http://dx.doi.org/10.21138/GF.558

Vinculados a la reforma del sistema tributario de 1845 , se desarrollarían los Amillaramientos a partir de 1850. Afectados por un elevado nivel de ocultación de datos, esta fuente fue progresivamente corregida y sustituida por el primer catastro, que se apoyaría en la representación gráfica, a través de mapas topográficos, para lograr una mayor veracidad en los datos (Pro, 1995; Vallejo, 2000). Los trabajos vinculados con el denominado "avance catastral" se iniciarían en 1870, tardando más de 100 años en concluirse, al tener que solventar diversos impedimentos durante su desarrollo (Pro, 1995; Vallejo, 2010).

En este progresivo incremento de las fuentes estadísticas apoyadas en cartografía, su temática y fiabilidad, en 1940 se hacía pública la orden por la que se encargaba al Consejo Agronómico la realización del primer Mapa Agronómico, Técnico y Comercial de Productos Agrícolas (1:50.000), cuyos trabajos serían el precursor del posterior Mapa de Cultivos y Aprovechamientos. El primero de estos mapas se publicaría en 1962 a escala 1:1.000.000, al que le seguiría, en 1974, mapas a escala 1:50.000 que se han consolidado como la base mediante la que se generalizan mapas provinciales (1:200.000) y la síntesis nacional (1:1.000.000) en 1988.

A finales del siglo XX llegaría la cartografía digital, siendo el mapa de usos y aprovechamientos una de las primeras fuentes que serían digitalizadas en aquel momento. Éstos serían los antecedentes que han servido de base al actual sistema informatizado, a través de Tecnologías de la Información Geográfica (TIG), en el que se desarrolla, entre otros, el Sistema de Información Geográfica de Datos Agrarios (SIGA) y el Sistema de Información Geográfica de Parcelas Agrícolas (SIGPAC) a escala nacional. Asimismo, también cabe desatacar otra fuente destinada al reconocimiento territorial, como es el Sistema de Ocupación del Suelo de España (SIOSE). Este sintetiza la delimitación de unidades territoriales por sus características biofísicas (coberturas o land cover), junto con las vinculadas a una funcionalidad o dedicación socioeconómica (usos o land use), dónde ambos (uso y cobertura) identifican la ocupación del suelo como reflejo último de diferentes procesos que confluyen en ese espacio, como pueden ser los vinculados al sistema agrario.

A nivel autonómico, Canarias, al igual que el resto de comunidades autónomas, se ha apoyado en las estadísticas desarrolladas a escala estatal, así como su representación cartográfica. A finales del siglo XX, la consideración de las particularidades de unos ámbitos insulares diversos y heterogéneos, en los que cada sistema agrario posee características propias, influidas por contextos socio-económicos y territoriales singulares, ha hecho que la escala de representación de estos mapas (1:50.000 y 1:25.000) no reflejara el nivel de detalle necesario para comprender esta especificidad. En este sentido, con el apoyo de las técnicas y tecnologías existentes en ese momento, sobre la recopilación y manejo de información geográfica en formato digital a mayor escala, se iniciarían las primeras campañas de recopilación de datos en campo para la elaboración del Mapa de Cultivos de Canarias, implementado en un Sistema de Información Geográfica (SIG) mediante trabajos que se extenderían entre 1998 y 2004.

A partir de esta primera experiencia, el SIG se posicionaría como soporte clave en la construcción de esta fuente. Así, actualmente cada una de las siete islas cuenta con más de un mapa de cultivos, implementado como base gráfica vectorial, a partir de diferentes campañas. Por otro lado, los aspectos conceptuales, técnicos y metodológicos que lo definen han evolucionado, a través de un necesario proceso de aprendizaje, en el que el saber hacer adquirido en torno a la abstracción 
García Cruz, J.I.; Febles Ramírez, M.F.; Pestana Pérez, G. (2017): “Las Tecnologías de la Información Geográfica aplicadas a la sistematización de los sistemas agrarios. Bases para el estudio de su dimensión espacial y temporal. El caso del mapa de cultivos de canarias (España)", GeoFocus (Artículos), $n^{\circ}$ 19, p. 181-206. ISSN: 1578-5157 http://dx.doi.org/10.21138/GF.558

de los sistemas agrarios de Canarias, ha permitido incorporar continuas mejoras que tratan de dar un mejor encaje de la fuente con la realidad territorial que trata de sistematizar. Como resultado, se ha generado un reconocimiento territorial adaptado a estas especificidades, diferenciado de los modelos de datos y metodologías implementadas en las fuentes destinadas al conjunto del estado, aportando una visión adaptada a estas particularidades.

En la actualidad, el Mapa de Cultivos de Canarias es una de las fuentes relevantes para entender los sistemas agrarios insulares, incrementando el nivel de detalle de la información oficial disponible, aportando una lectura más completa y detallada de cada territorio insular, aunque para ello debemos tomar en cuenta una serie de aspectos clave respecto a su construcción y su relación con otras estadísticas agrarias o fuentes cartográficas, sobre todo entendiendo que no existe una correspondencia en sus datos al utilizar diferentes métodos de observación y medición (García Rodríguez, 2015; Évora, 2015). Aunque técnicamente es factible el establecimiento de una leyenda común entre todas ellas, esta cuestión sigue sin estar resuelta, a lo hay que añadir que cada método de medición (encuesta, observación directa en campo, etc.) no suele coincidir en las cifras sobre la superficie de un mismo cultivo.

\section{Las particularidades del contexto territorial de Canarias}

En su condición del archipiélago, con una extensión de $7.493 \mathrm{~km}^{2}$, el 1,5\% de la superficie nacional, la comunidad autónoma de Canarias se caracteriza por ser un territorio insular fragmentado (ver figura 1).

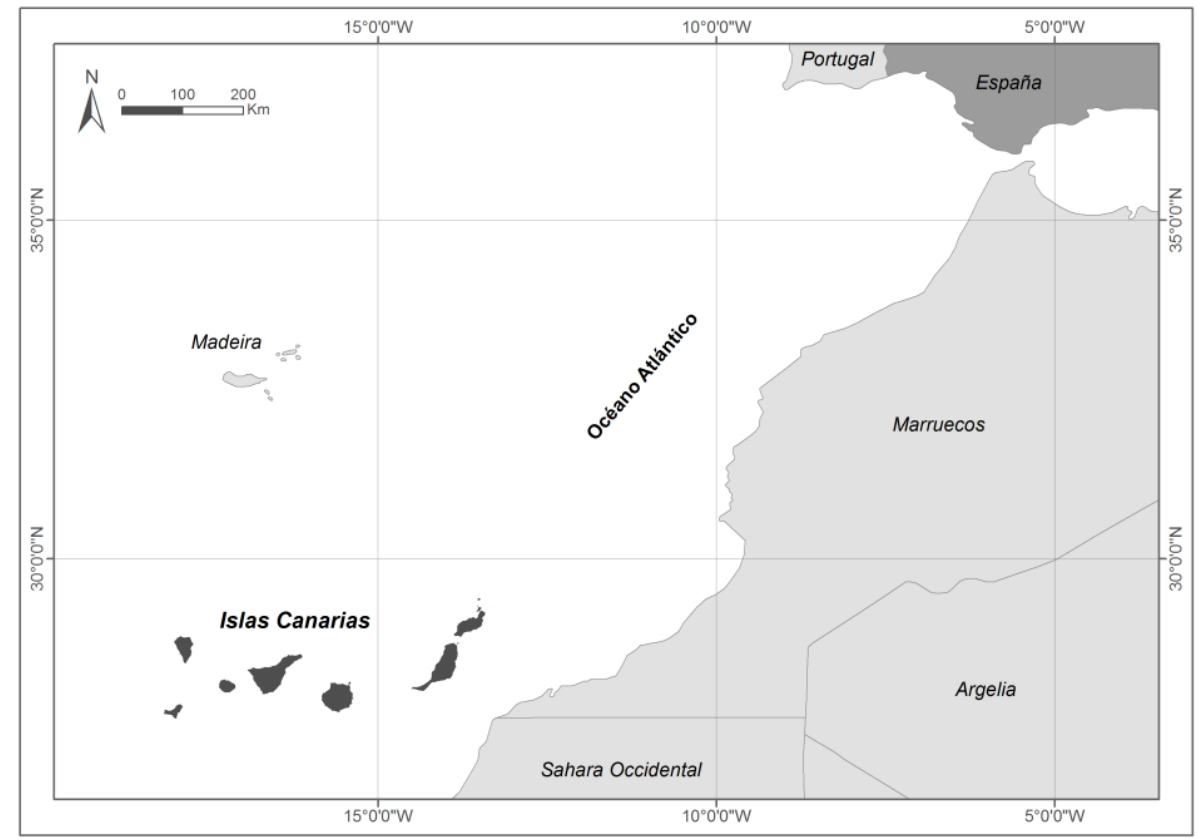

Figura 1. Localización del archipiélago Canario.

Fuentes: Diva-Gis Free Data. Grafcan. Elaboración propia. 
García Cruz, J.I.; Febles Ramírez, M.F.; Pestana Pérez, G. (2017): "Las Tecnologías de la Información Geográfica aplicadas a la sistematización de los sistemas agrarios. Bases para el estudio de su dimensión espacial y temporal. El caso del mapa de cultivos de canarias (España)", GeoFocus (Artículos), $n^{\circ}$ 19, p. 181-206. ISSN: 1578-5157 http://dx.doi.org/10.21138/GF.558

Cada isla presenta una serie de particularidades derivadas, entre otros factores, de su relieve, lo que condiciona la existencia de diferentes microclimas o topoclimas, que a su vez se expresan en una distribución altitudinal diferenciada por vertientes de distintas formaciones vegetales (pisos de vegetación). Éstas se reparten en una diversidad de paisajes, en los que predominan superficies de elevada pendiente, condicionado por su carácter discontinuo, afectado por una red de barrancos y la presencia de estructuras volcánicas (conos, roques, malpaíses, etc.), entre otros elementos (ver tabla 1).

Desde el punto de vista humano, la superficie urbanizada supone actualmente el $6 \%$ regional (García Cruz, 2013), frente a un 5,8 \% bajo cultivo activo (Pestana, Febles y de la Rosa, 2015). Los cambios producidos en los modelos y ejes de desarrollo económico, entre finales del siglo XX y principios del XXI, propiciaron una progresiva pérdida de peso del modelo agropecuario frente a la terciarización del modelo económico, sintetizado este en la pérdida de un uso activo de las zonas de montaña, la reconversión de la medianía, así como el desplazamiento de la presión urbanizadora hacia el litoral (Hernández et.al., 2010; García Cruz, 2013).

Tabla 1. Caracterización insular

\begin{tabular}{|r|r|r|r|r|r|r|}
\hline \multicolumn{1}{|c|}{ Isla } & $\begin{array}{c}\text { Superficie } \\
\text { total }^{\mathrm{a}}\end{array}$ & $\begin{array}{c}\text { Superficie } \\
\text { islotes y } \\
\text { roques }^{\mathrm{a}}\end{array}$ & $\begin{array}{c}\text { Superficie } \\
\text { protegida }^{\mathrm{b}}\end{array}$ & $\begin{array}{c}\text { Superficie } \\
\text { urbanizada }^{\mathrm{c}}\end{array}$ & $\begin{array}{c}\text { Sup. }> \\
1200 \mathrm{~m} \text { de } \\
\text { altitud }^{\mathrm{d}}\end{array}$ & $\begin{array}{c}\text { Sup. }>30 \% \\
\text { de pendiente }^{\mathrm{d}}\end{array}$ \\
\hline Lanzarote & 886,8 & 40,9 & 356,8 & 47,0 & 0 & 130,4 \\
\hline Fuerteventura & $1.664,3$ & 4,6 & 487,7 & 55,5 & 0 & 429,2 \\
\hline Gran Canaria & $1.560,2$ & 0,1 & 786,1 & 163,4 & 114,1 & 942,3 \\
\hline Tenerife & $2.034,6$ & 0,2 & 996,8 & 160,2 & 589,6 & $1.004,1$ \\
\hline La Gomera & 369,8 & 0,1 & 191,7 & 7,5 & 11,9 & 297,3 \\
\hline La Palma & 708,5 & 0,2 & 374,7 & 21,6 & 172,6 & 488,2 \\
\hline El Hierro & 268,8 & 0,1 & 153,5 & 7,0 & 17,2 & 144,5 \\
\hline Canarias & $7.493,1$ & 46,2 & $3.347,2$ & 462,2 & 905,5 & $3.436,1$ \\
\hline
\end{tabular}

Unidades: Kilómetros cuadrados $\left(\mathrm{km}^{2}\right)$

a. ISTAC (2008). b. Resultado de la superposición de la superficie terrestre afectada por la Red Canaria de Espacios Naturales Protegidos (ENP) y Zonas Especiales de Conservación (ZEC) de la Red Natura 2000. c.

García Cruz (2013). d. Elaboración propia a través de los Modelos Digitales de Terreno generados con tecnología Lidar (GRAFCAN, 2010-2011).

Fuentes: ISTAC (2008), Consejería de Obras Públicas, Transportes y Política Territorial, GRAFCAN (20102011), García Cruz (2013).

Considerando los datos aportados por los anuarios de estadística agraria del Ministerio de Agricultura, Alimentación y Medio Ambiente, el progresivo incremento de la superficie agraria entre principios y mediados del siglo XX (de las 44.300 ha en 1921 a las 175.300 ha en 1960), condicionado por hitos tan importantes como el periodo de autarquía en la postguerra, se invertiría a partir de la década de los sesenta, con la recuperación económica vinculada al cambio en el modelo socio-económico. Así, esta progresiva reducción llevaría de las 141.500 ha en 1980 a las actuales 
García Cruz, J.I.; Febles Ramírez, M.F.; Pestana Pérez, G. (2017): “Las Tecnologías de la Información Geográfica aplicadas a la sistematización de los sistemas agrarios. Bases para el estudio de su dimensión espacial y temporal. El caso del mapa de cultivos de canarias (España)", GeoFocus (Artículos), no 19, p. 181-206. ISSN: 1578-5157 http://dx.doi.org/10.21138/GF.558

41.412,72 ha (Pestana et al., 2015). Con todo ello, la mayor pérdida de superficie se sitúa entre los años sesenta y noventa (Fernández-Palacios et al., 2004), reflejadas igualmente en una considerable reducción del empleo en el sector, pasando del $54 \%$ de la población activa a mediados de siglo XX (Fernández-Palacios et al., 2004) al 2,7 \% actual (ISTAC, 2016).

En términos globales, los sistemas agrarios suponen actualmente en Canarias el 18,41\% de la superficie regional, limitándose al 5,58 \% si sólo se contabiliza la cultivada de forma activa. A esto hay que sumar las particularidades de su distribución en el archipiélago, vinculadas a cada contexto insular en su extensión, distribución y predominio de determinadas tipologías de cultivo, entre otros aspectos.

Cada isla presenta particularidades derivadas de su contexto físico (relieve, clima, hidrología y vegetación) y de la interrelación con aspectos culturales, expresados en el desarrollo de técnicas y formas de cultivo propias (enarenado, jable, gavias, etc.), periodos, sistemas de rotación, entre otros. Sumándole la evolución histórica y el contexto socio-económico, los sistemas agrarios han ido experimentando cambios a lo largo del tiempo. Estos cambios se reflejan, entre otros aspectos, en la aparición, desaparición, pérdida de peso, incremento o cambio de determinados cultivos que, a su vez, pueden tener un carácter intensivo, estar enfocados a la exportación, consumo interno o autoconsumo.

La suma de todos estos elementos condiciona una elevada complejidad, inherente a cada contexto insular, que limita la capacidad de las fuentes de información desarrolladas a nivel estatal a la hora de medirlos y caracterizarlos. En este sentido, con una escala de detalle a 1:50.000 o 1:25.000, la identificación y representación gráfica de estas realidades insulares resulta muy limitada, lo que ha demandado el planteamiento y desarrollo de una fuente adaptada a estas realidades, como es el Mapa de Cultivos de Canarias, desarrollado a escala 1:2.500, mediante una metodología que trata de acomodarse a las singularidades de las islas (ver Figura 2). 
García Cruz, J.I.; Febles Ramírez, M.F.; Pestana Pérez, G. (2017): “Las Tecnologías de la Información Geográfica aplicadas a la sistematización de los sistemas agrarios. Bases para el estudio de su dimensión espacial y temporal. El caso del mapa de cultivos de canarias (España)", GeoFocus (Artículos), $n^{\circ}$ 19, p. 181-206. ISSN: 1578-5157 http://dx.doi.org/10.21138/GF.558

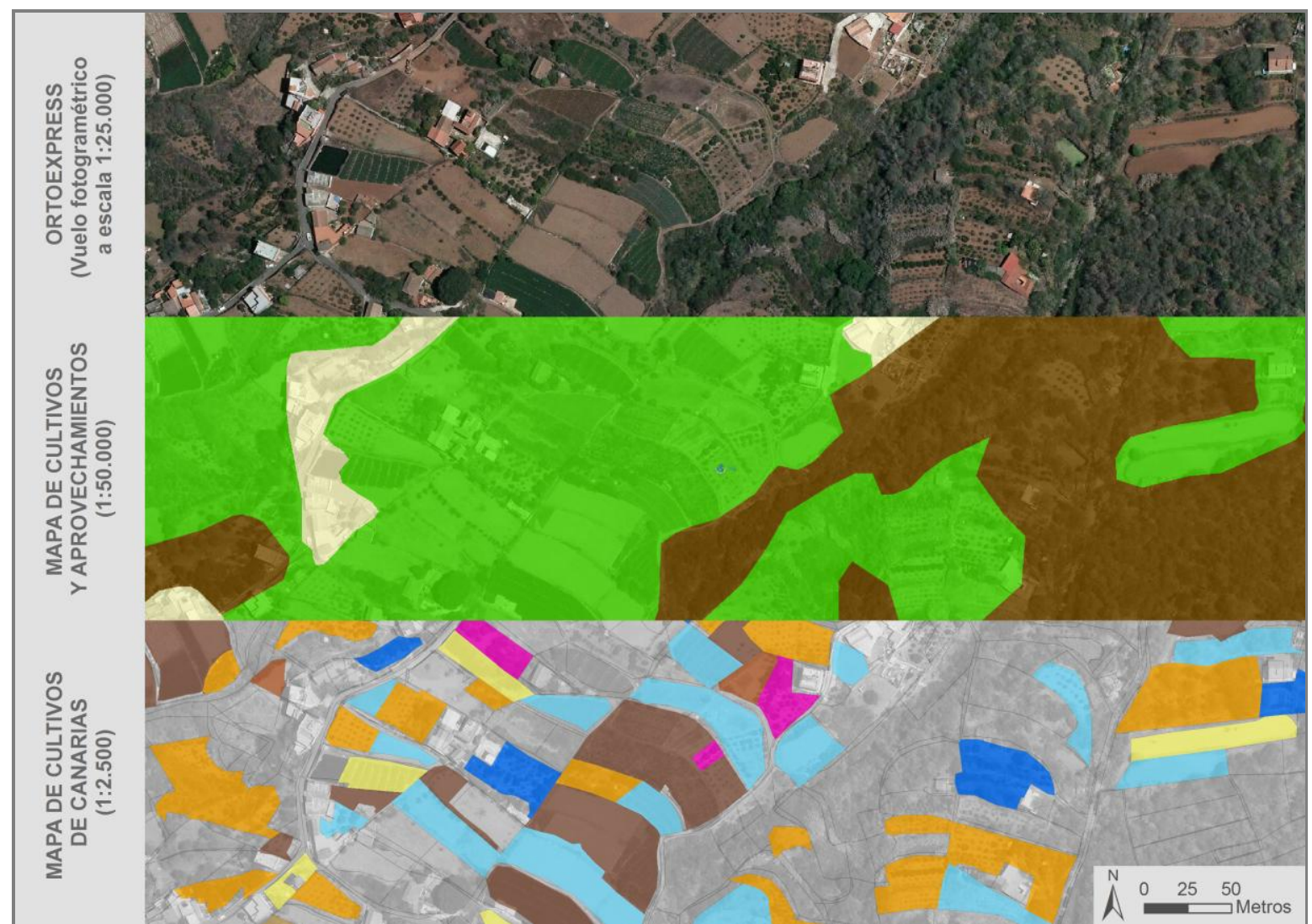

Figura 2. Comparativa del nivel de detalle entre el Mapa de Cultivos y Aprovechamientos y el Mapa de Cultivos de Canarias.

Fuentes: Mapa de cultivos y aprovechamientos (Ministerio de Agricultura, Alimentación y Medio Ambiente. Gobierno de España). Mapa de cultivos de Canarias (Consejería de Agricultura, Ganadería, Pesca y Aguas. Gobierno de Canarias). Ortoexpress, 2012 (Grafcan).

Fuente: Elaboración propia.

\section{El proceso de elaboración del Mapa de Cultivos de Canarias sobre la base de las TIG}

De forma global, las TIG son la suma de diferentes tecnologías que emplean una diversidad de técnicas y métodos en el manejo de información georeferenciada o geoinformación, lo que facilita una dimensión espacial de su análisis. Así, las TIG agrupan a la Cartografía y la Fotointerpretación, como elementos tradicionales, a los que se le han unido la Teledetección, los Sistemas de Posicionamiento Global y los Sistemas de Información Geográfica (SIG), como elementos modernos vinculados con el desarrollo tecnológico. Del mismo modo, se asocian a una nueva conceptualización del territorio, planteando nuevas formas de observación, análisis e intervención (Buzai y Baxendale, 2006).

Dado el marcado carácter territorial de los sistemas agrarios, es lógica y consecuente su consideración como una variable espacial que puede ser medida y caracterizada mediante el uso de geoinformación. En este sentido, ésta es definida como: "aquella que permite modelizar y representar los fenómenos geográficos o entidades del mundo real que configuran el amplio y 
García Cruz, J.I.; Febles Ramírez, M.F.; Pestana Pérez, G. (2017): “Las Tecnologías de la Información Geográfica aplicadas a la sistematización de los sistemas agrarios. Bases para el estudio de su dimensión espacial y temporal. El caso del mapa de cultivos de canarias (España)", GeoFocus (Artículos), no 19, p. 181-206. ISSN: 1578-5157 http://dx.doi.org/10.21138/GF.558

variado espacio en el que se desarrolla la actividad humana: el territorio" (Zurita, 2011, 39). Por esta razón, se considera una base válida para la construcción de nuevas fuentes que vinculan la información captable sobre los objetos (atributos), con su localización y dimensión espacial (lugar y forma), añadiendo la posibilidad de analizar sus relaciones (vinculaciones espaciales), lo que facilita la caracterización del sistema agrario.

Todo ello supone trasladar una compleja realidad a un modelo digital que la simplifica mediante un proceso de abstracción apoyado en el uso combinado de la percepción de la realidad con diferentes instrumentos de medición (Bosque, 1999). Éste se basa en el reconocimiento y delimitación de los objetos como una figura geométrica (modelo vectorial) o un conjunto de celdas (modelo raster), al que se le vinculan un atributo (modelo raster) o múltiples (modelo vectorial). Su carácter espacial permite que esta base de datos gráfica pueda considerar las relaciones existentes entre los objetos (relaciones topológicas) mediante la localización, distribución, asociación, interacción y evolución (Buzai, 2010), así como entre los objetos y sus atributos. Como resultado, la realidad territorial pasa a convertirse en una representación abstracta, a través de un modelo informático que la descompone y estratifica en función de múltiples temáticas que se interrelacionan de forma espacial (Gómez y Barredo, 2005), no exenta de ciertas limitaciones en la consideración, únicamente, de variables localizables y medibles (Tapiador, 2006) que deben ser consideradas a la hora de valorar sus resultados, dado que éstos podrían ser parciales ante la no consideración de otras variables a-espaciales (Dobson, 1983, 1993; Capel, 2005 y 2009).

Así, las TIG facilitan las bases tecnológicas, metodológicas y conceptuales necesarias para la implementación de sistemas de información que abstraigan la realidad territorial de una forma ordenada y coherente. Esto supone desarrollar la obtención de conocimiento contrastable mediante la medición sistemática de diferentes variables espaciales (Tapiador, 2006) que permitan entender los procesos territoriales con plasmación espacial (localizables y medibles).

Dentro de esta tecnología, el desarrollo del Mapa de Cultivos de Canarias se ha apoyado principalmente en los Sistemas de Información Geográfica (SIG) ${ }^{2}$. En este sentido, considerando la amplia muestra de trabajos que han entrado en los aspectos conceptuales, técnicos y metodológicos vinculados al planteamiento e implementación de un SIG (Bosque, 1992; Gutiérrez, 2000; Gómez y Barredo, 2005; Tomlinson, 2007; Buzai y Baxendale, 2006; Buzai, 2010; Tapiador, 2006; Zurita, 2011), partimos de la necesaria consideración de un proceso ordenado y coherente (Buzai, Baxendale y Cruz, 2009). En términos globales, a la hora de plantear el diseño e implementar un SIG, "hablamos de la combinación de hardware, software, datos, técnicas, procedimientos y usuarios, para poder adquirir, almacenar, editar, analizar y publicar información geográfica, con el fin de dar respuesta a problemas complejos de carácter territorial, con la posibilidad de que ésta pueda ser desarrollada desde un punto de vista multidisciplinar, donde su uso desarrolla una constante evolución de sus capacidades" (García Cruz, 2015, 221).

Con todo ello, el diseño original del Mapa de Cultivos de Canarias, su implementación y posterior incorporación de mejoras, se ha visto condicionado por tres elementos clave.

2 Cabe señalar que actualmente se desarrollan trabajos que buscan la introducción de técnicas de teledetección, a través de Lidar, para el reconocimiento de cultivos, así como los trabajos de campo se ven apoyados por sistemas de posicionamiento (GPS), entre otros aspectos, por lo que el uso de las TIG es más amplio, aunque predomine el papel del SIG. 
García Cruz, J.I.; Febles Ramírez, M.F.; Pestana Pérez, G. (2017): "Las Tecnologías de la Información Geográfica aplicadas a la sistematización de los sistemas agrarios. Bases para el estudio de su dimensión espacial y temporal. El caso del mapa de cultivos de canarias (España)", GeoFocus (Artículos), $n^{\circ}$ 19, p. 181-206. ISSN: 1578-5157 http://dx.doi.org/10.21138/GF.558

En primer lugar, considerando lo anteriormente expuesto, cada contexto insular posee elementos particulares que dificultan la homogeneización de determinados aspectos a escala regional (ej. técnicas como el jable y el enarenado tienen mayor peso en unas islas frente a otras donde es casi testimonial). En segundo lugar, la recopilación de datos en campo se ve sujeta a un sistema agrícola complejo y dinámico, en el que la interacción de múltiples tipos de cultivos, periodos de cosecha, asociaciones, sucesiones anuales, entre otros aspectos, dificulta la planificación de los trabajos de campo (ej. existen cultivos que no son reconocibles hasta determinada fase de crecimiento). Por último, esta compleja y dinámica realidad debe ser abstraída mediante un modelo de datos informático (en este caso vectorial), con las potencialidades y limitaciones que éste ofrece a la hora sistematizar la variable territorial considerada. Con ello, cada uso es localizado y delimitado a través de un recinto (elemento vectorial), asignándole una serie de atributos que codifican las características que precisan ser recopiladas sobre éste (tipo de cultivo, existencia de invernadero o regadío, superficie, etc.), dando lugar a una base de datos gráfica en la que se concentra una abstracción de la realidad territorial.

Resolver estos tres aspectos ha supuesto la aplicación de un conjunto significativo de decisiones técnicas a lo largo de cada una de las campañas. La base gráfica se ha desarrollado en cada contexto insular, según las posibilidades presupuestarias, con un diseño que ha ido evolucionando con el proceso de aprendizaje, enriqueciendo su metodología, ajustando sus criterios técnicos y mejorando sus fuentes cartográficas de base. Con todo ello, actualmente los trabajos de campo coinciden con el año agrícola, planificando la recogida de datos en función de los momentos más óptimos para identificar cada cultivo, desarrollando una clasificación adaptada a la diversidad ya reconocida en anteriores campañas, así como se han incorporado aspectos vinculados con la presencia del regadío, entre otros elementos.

Como resultado, en la actualidad se cuenta con un histórico de diferentes campañas por islas que ha permitido dar un nuevo paso en su desarrollo, planteando la variable temporal como nuevo aspecto que permita identificar y medir su evolución reciente.

Cada campaña parte de la elección del año agrícola en el que se van a llevar a cabo los trabajos de campo, por lo que el mapa resultante se constituye en una foto fija condicionada por el momento en el que se produce la observación. Para ello, la administración debe gestionar aspectos tan diversos como los presupuestarios, administrativos, técnicos o recursos humanos, con la suficiente antelación como para que los trabajos encajen con el año agrícola de la isla que corresponda en cada campaña o se aproximen, en la medida de lo posible, a éste, cuestión que sigue perfeccionándose con el objetivo de reducir el periodo de tiempo entre campañas en cada espacio insular.

La planificación de los trabajos comienza con una zonificación de la isla en función de sus características físicas (altitud, orientación, etc.), los cultivos predominantes, su periodicidad o sistema de rotación y algunos otros aspectos secundarios. Cabe indicar que la extensión de cada unidad se vincula con el tiempo necesario para su procesamiento en campo y posterior revisión en gabinete, considerando el margen de tiempo suficiente como para poder realizar las correcciones oportunas para su cierre, antes de que los cultivos que engloba cambien dentro de su dinámica anual. Así, la observación de cada tipología depende de si ésta es permanente, como pueden ser la platanera y diferentes frutales, o estacional (plurianual o rotacional), siendo reconocible a partir de un determinado momento de su fase de crecimiento o sujeto a la complejidad de la rotación anual. 
García Cruz, J.I.; Febles Ramírez, M.F.; Pestana Pérez, G. (2017): “Las Tecnologías de la Información Geográfica aplicadas a la sistematización de los sistemas agrarios. Bases para el estudio de su dimensión espacial y temporal. El caso del mapa de cultivos de canarias (España)", GeoFocus (Artículos), $n^{\circ}$ 19, p. 181-206. ISSN: 1578-5157 http://dx.doi.org/10.21138/GF.558

Tomando en cuenta la heterogeneidad de algunas zonas, la recopilación de datos no puede evitar el desajuste que puede existir ante la no presencia de determinados cultivos en el momento de la observación, por lo que la fuente no deja de ser una aproximación a una realidad territorial compleja, heterogénea y dinámica (ver Figura 3).

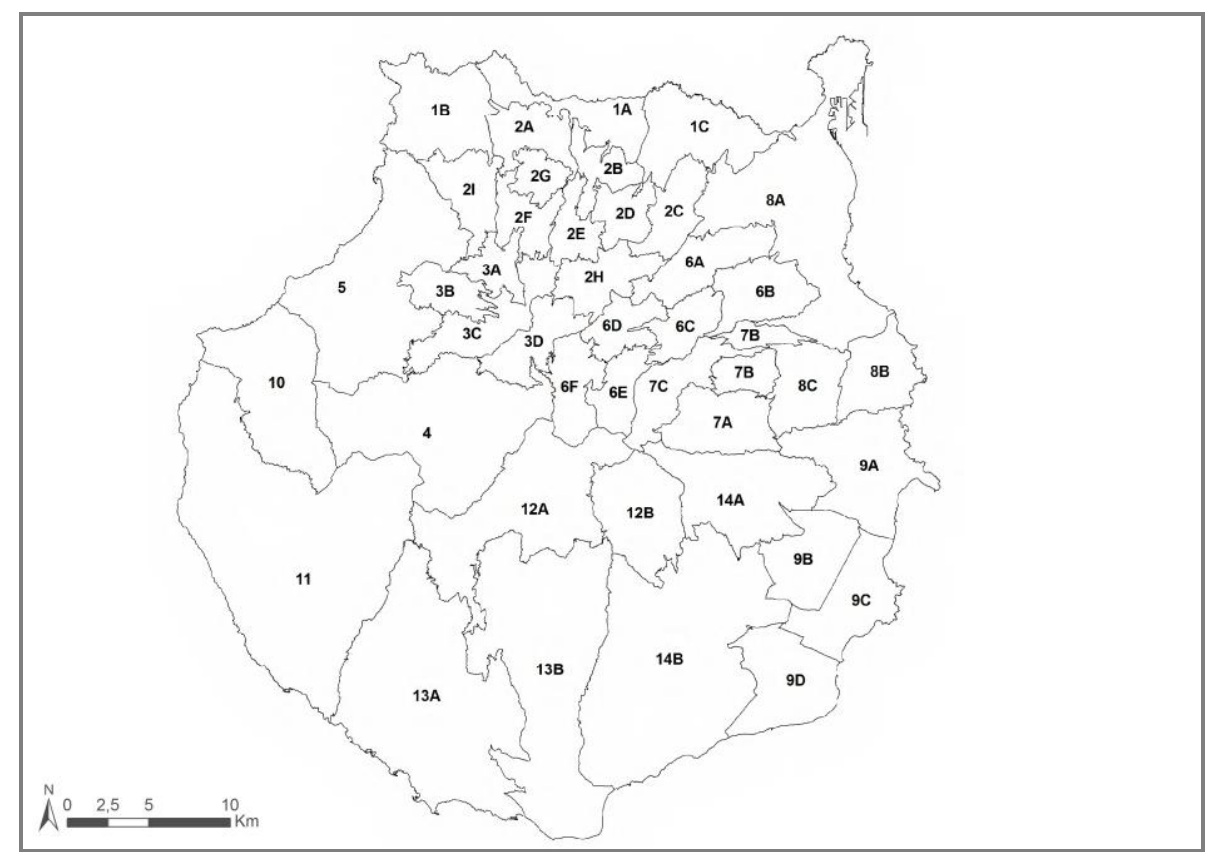

Figura 3. Ejemplo de la zonificación realizada para los trabajos de campo del mapa de cultivos de Gran Canaria (Campaña 2013).

Fuente: Servicio de planificación de obras y ordenación rural. Dirección general de agricultura y desarrollo rural. Consejería de Agricultura, Ganadería, Pesca y Aguas. Gobierno de Canarias.

Se deben incorporar otras dificultades como las condiciones climáticas que predominan en cada campaña, dado que el retraso de las lluvias invernales, veranos con temperaturas elevadas, los efectos de algún temporal, la sucesión de periodos de calima, entre otras situaciones, pueden impedir, retrasar, cambiar o dañar el desarrollo de alguno o varios tipos de cultivo según la zona, lo que obliga al replanteamiento de los trabajos de campo cuando éstos ya se están llevando a cabo.

Del mismo modo, cambios puntuales en el modelo socio-económico a escala insular pueden no ser considerados por la fuente en un primer momento. Al depender de los años en los que se realizan las campañas, existe un margen de 6 a 14 años entre cada una dependiendo de la isla y su dinamismo, por lo que procesos, como puede ser la posible recuperación de fincas agrícolas en Tenerife tras el inicio de la crisis económica, no pueden reflejarse en los datos hasta poder contrastarse la campaña disponible (2008) con la que se lleve a cabo posteriormente (2016), cuestión que pretende mejorase con la reducción de tiempos entre campañas.

Programado el trabajo de campo, el proceso de inventario se estructura en torno a dos operaciones básicas, esto es, la delimitación perimetral del cultivo (recinto) y la recopilación de los atributos asociables (información alfanumérica). 
García Cruz, J.I.; Febles Ramírez, M.F.; Pestana Pérez, G. (2017): “Las Tecnologías de la Información Geográfica aplicadas a la sistematización de los sistemas agrarios. Bases para el estudio de su dimensión espacial y temporal. El caso del mapa de cultivos de canarias (España)", GeoFocus (Artículos), $n^{\circ}$ 19, p. 181-206. ISSN: 1578-5157 http://dx.doi.org/10.21138/GF.558

Este procedimiento se desarrolla con el apoyo de equipos informáticos (PDA, tablets o portátiles con pantalla táctil), sistemas de posicionamiento (GPS) y un aplicativo desarrollado de forma específica para facilitar el trabajo de dibujo y recopilación de datos mediante la adaptación de software libre (ver Figura 4). Todo este material es aportado a una serie de cuadrillas previamente formadas y puestas a prueba en zonas piloto, que desarrollan su trabajo siguiendo un manual de instrucciones sobre delimitación y reconocimiento. Como elementos adicionales, cada equipo se dota de un vehículo todoterreno, prismáticos y cámaras fotográficas que facilitan el desarrollo y seguimiento de los trabajos, así como contacto con el equipo de gestión en gabinete. Este último se responsabiliza de la reorganización del trabajo de campo cuando es necesario, prestar labores de apoyo y asesoramiento, así como aplicar los controles de calidad y coherencia de la información.

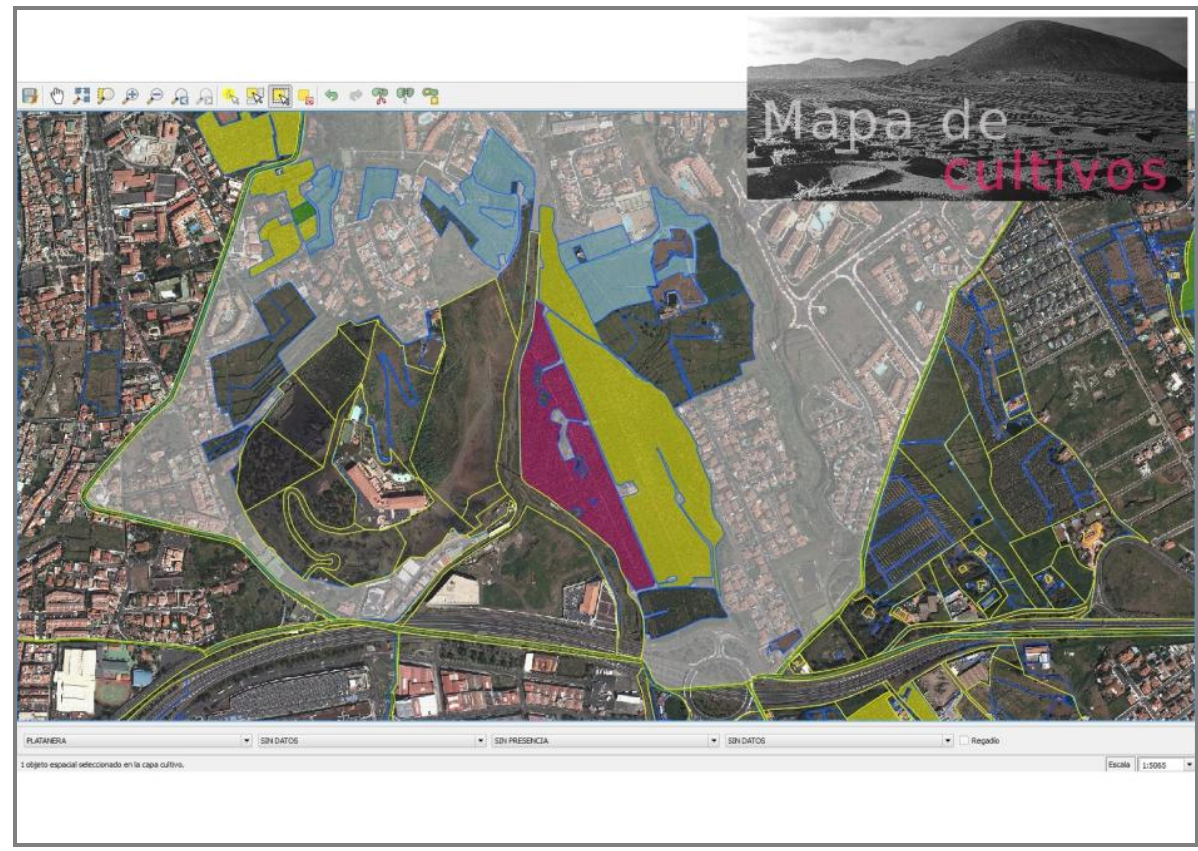

Figura 4. Ejemplo de la versión adaptada del software Qgis para el trabajo de campo.

Fuente: Servicio de planificación de obras y ordenación rural. Dirección general de agricultura y desarrollo rural. Consejería de agricultura, ganadería, pesca y aguas. Gobierno de Canarias.

En primer lugar, la delimitación territorial del denominado "recinto" responde al objetivo de distinguir éste de la consideración de parcela catastral o finca registral, a fin de centrarse en su aspecto agronómico (reconocimiento objetivo de un uso agrícola como unidad de producción). Cabe indicar que se trata de unidades no vinculadas con la titularidad real del terreno, por lo que una misma propiedad puede estar afectada por uno o varios recintos que no responden necesariamente a sus límites. Aun así, el trabajo de campo se sustenta en la fotografía aérea (ortofotografía u ortoexpress) y recintos aportados por el SIGPAC nacional, por lo que el dibujo de cada uno puede apoyarse en el trazado ya existente en la cartografía base o modificarlo en función del encaje con el uso agrícola que pretende delimitar. Del mismo modo, al trabajar con la unidad de mayor desagregación (unidad de producción), la fuente no permite generalizar las explotaciones agrícolas a las que se vinculan por si misma (cuestión que puede salvarse mediante la superposición de otras bases gráficas, como el catastro o el registro de la propiedad). Esto es, no es posible saber cuántas 
García Cruz, J.I.; Febles Ramírez, M.F.; Pestana Pérez, G. (2017): "Las Tecnologías de la Información Geográfica aplicadas a la sistematización de los sistemas agrarios. Bases para el estudio de su dimensión espacial y temporal. El caso del mapa de cultivos de canarias (España)", GeoFocus (Artículos), no 19, p. 181-206. ISSN: 1578-5157 http://dx.doi.org/10.21138/GF.558

unidades de producción tiene una determinada explotación agrícola, así como estas unidades no tienen por qué ser coincidentes con la propiedad, al menos que el Mapa de Cultivo se cruce con otras bases gráficas que permitan añadir esta posibilidad.

Tabla 2. Distribución de categorías, agrupaciones y cultivos

\begin{tabular}{|c|c|c|c|c|c|}
\hline Categoría & Agrupación & Cultivo & Categoría & Agrupación & Cultivo \\
\hline \multirow{18}{*}{ Frutales } & Cítricos & Cítricos & \multirow{2}{*}{ Huerta } & Huerto familiar & Huerto familiar \\
\hline & \multirow{10}{*}{$\begin{array}{c}\text { Frutales } \\
\text { Subtropicales }\end{array}$} & Aguacate & & Papa & Papa \\
\hline & & Aloe & \multirow{5}{*}{ Ornamentales } & \multirow{5}{*}{$\begin{array}{l}\text { Ornamentales y } \\
\text { aromáticas }\end{array}$} & Aromáticas \\
\hline & & Mango & & & Ornamentales \\
\hline & & Moringa & & & Próteas \\
\hline & & Papaya & & & Strelitzias \\
\hline & & Parchita & & & Viveros \\
\hline & & Piña & \multirow{6}{*}{ Otros } & \multirow{3}{*}{$\begin{array}{l}\text { Cereales y } \\
\text { leguminosas }\end{array}$} & Cereal varios \\
\hline & & Pitaya & & & Leguminosas \\
\hline & & Subtropicales varios & & & Millo \\
\hline & & Tunera & & \multirow{3}{*}{$\begin{array}{c}\text { Otras } \\
\text { asociaciones }\end{array}$} & A. Cítricos-Hortalizas \\
\hline & \multirow{7}{*}{$\begin{array}{c}\text { Frutales } \\
\text { Templados }\end{array}$} & Almendro & & & $\begin{array}{l}\text { A. Cítricos- } \\
\text { Ornamentales }\end{array}$ \\
\hline & & Granado & & & A. Cítricos-Papa \\
\hline & & Higuera & \multirow{2}{*}{ Platanera } & \multirow{2}{*}{ Platanera } & A. Platanera-Cítricos \\
\hline & & Olivo & & & Platanera \\
\hline & & Templado hueso & Tomate & Tomate & Tomate \\
\hline & & Templado pepita & \multirow{8}{*}{ Viña } & \multirow{6}{*}{ A. Viña-Otros } & A. Viña-Barbecho \\
\hline & & Templados varios & & & A. Viña-Cítricos \\
\hline \multirow{13}{*}{ Huerta } & \multirow{2}{*}{$\begin{array}{c}\text { A. Templados- } \\
\text { Papa }\end{array}$} & A. Templados-Millo & & & A. Viña-Hortalizas \\
\hline & & A. Templados-Papa & & & A. Viña-Huerta Limpia \\
\hline & \multirow{2}{*}{ Barbecho } & Barbecho & & & A. Viña-Templados \\
\hline & & Huerta limpia & & & A. Viña-Tunera \\
\hline & \multirow{9}{*}{ Hortalizas } & Batata & & A. Viña-Papa & A. Viña-Papa \\
\hline & & Cebolla & & Viña & Viña \\
\hline & & Fresa & \multirow{2}{*}{ Pastizal } & \multirow{2}{*}{ Pastos } & Pastizal \\
\hline & & Hortalizas varias & & & Tagasaste \\
\hline & & Melón-Sandia & \multirow{2}{*}{ Sin cultivo } & \multirow{2}{*}{ Sin Cultivo } & Abandono reciente \\
\hline & & Name & & & Abandono prolongado \\
\hline & & Pepino & \multirow{2}{*}{$\begin{array}{c}\text { Resto de } \\
\text { superficies }\end{array}$} & Erial & Erial \\
\hline & & Caña de azúcar & & Monte & Monte \\
\hline & & Tabaco & & & \\
\hline
\end{tabular}

Fuente: Mapa de cultivos de Canarias. Elaboración propia. 
García Cruz, J.I.; Febles Ramírez, M.F.; Pestana Pérez, G. (2017): "Las Tecnologías de la Información Geográfica aplicadas a la sistematización de los sistemas agrarios. Bases para el estudio de su dimensión espacial y temporal. El caso del mapa de cultivos de canarias (España)", GeoFocus (Artículos), no 19, p. 181-206. ISSN: 1578-5157 http://dx.doi.org/10.21138/GF.558

En segundo lugar, dada la diversidad de cultivos existentes, se ha planteado una clasificación en función de aquellos grupos con mayor relevancia (presencia y superficie). Este listado ha ido evolucionando en la medida que las diferentes campañas han evidenciado la aparición de nuevas tipologías a considerar, como el olivo o el aloe, lo que ha condicionado la medición de cambios entre las diferentes campañas. Con todo ello, en este momento se trabaja con 10 grandes categorías, divididos en 20 agrupaciones, que aglutinan 61 tipos de cultivos $^{3}$ (ver tabla 2).

Lejos de resultar una operación sencilla, el carácter complejo, heterogéneo y dinámico de cada sistema agrario insular hace que una misma unidad de producción presente una combinación de cultivos de difícil separación, llegando a combinar la presencia de anuales con plurianuales y rotacionales. Por esta razón, dentro de los criterios a emplear a la hora de clasificar los cultivos, se han considerado una serie de particularidades que tratan de salvar esta cuestión.

En aquellos recintos en los que se reconoce más de un cultivo con difícil apreciación de su separación física, con una presencia similar o con una entidad suficiente como para no determinar el predominio de uno de ellos, se han establecido un conjunto de tipologías denominadas asociaciones (ej. Viña-papa). Por otro lado, la detección de cultivos en el borde de recintos se distingue en un campo aparte, identificando la presencia de templados, cítricos, subtropicales o viña en su perímetro (borde). En el caso particular de la viña, se distingue si ésta ocupa una parte o la totalidad de dicho perímetro $(25 \%, 50 \%, 75 \%, 100 \%)$.

Por último, aquellos cultivos que se encuentran de forma diseminada en el recinto, sosteniendo una determinada entidad en el mismo, se codifican de forma paralela indicando la presencia de un cultivo diseminado y la tipología que corresponda (ej. presencia de algunos almendros en un recinto con otro cultivo predominante). Así, cabe indicar que, ante la presencia aislada de un determinado elemento (ej. una higuera), su carácter marginal o aislado hace que no sea tomado en cuenta en el proceso de inventario. De este modo, sólo se consideran aquellos cultivos que responden al criterio de conformar un recinto con suficiente entidad territorial o poseer cierto peso dentro de este, lo que descarta elementos puntuales.

Con todo ello, la presencia de estas situaciones provoca un desajuste entre los datos alfanuméricos recopilados y su representación gráfica a través del mapa. Así, la clasificación de cultivos predominantes y asociaciones puede ser representada a través de éste, mientras que los cultivos de borde quedan supeditados a los cálculos aproximados que pueden realizarse sobre la tabla de atributos para estimar la superficie que restan a los principales. Asimismo, aquellos identificados como dispersos no pueden tener una representación gráfica real de su superficie, así como no es posible un cálculo acertado de ésta, por lo que sólo se identifica su presencia.

Como últimos puntos en esta metodología, los datos recopilados en campo son trasmitidos progresivamente para la comprobación de coherencia en gabinete. Este procedimiento se inicia con la comprobación visual de cada recinto sobre la última fotografía aérea disponible, realizando ajustes sobre los datos obtenidos en campo. En aquellos casos sobre los que aún pueda quedar duda, se programan y realizan nuevas salidas para la resolución "in-situ".

\footnotetext{
${ }^{3}$ Ver Pestana, Febles y de la Rosa [coord.] (2015).
} 
García Cruz, J.I.; Febles Ramírez, M.F.; Pestana Pérez, G. (2017): “Las Tecnologías de la Información Geográfica aplicadas a la sistematización de los sistemas agrarios. Bases para el estudio de su dimensión espacial y temporal. El caso del mapa de cultivos de canarias (España)", GeoFocus (Artículos), $n^{\circ}$ 19, p. 181-206. ISSN: 1578-5157 http://dx.doi.org/10.21138/GF.558

Tras el cierre definitivo del proceso de captura de datos, comprobación de coherencia y corrección, se procede al ajuste de los aspectos topológicos (corrección de posibles errores en la geometría del modelo vectorial), así como otros aspectos vinculados con la asignación de códigos de identificación únicos para cada recinto y otros campos que completan la base de datos gráfica.

Finalmente, tras aproximadamente un año de trabajo, dependiendo de cada isla, la campaña se da por concluida, procediendo al cálculo y publicación de las tablas de distribución de superficies en el portal web de la consejería competente (http://www.gobiernodecanarias.org/agricultura/agricultura/temas/mapa_cultivos). Del mismo modo, se facilita su difusión a través de la Infraestructura de Datos Espaciales de Canarias (IDECAN: http://www.idecanarias.es/idecan/), como elemento incorporado en su visor y servicio Web Map Service (WMS) disponible en su catálogo, aparte de la posibilidad de obtener una copia de la base gráfica, en formato shapefile, mediante solicitud por escrito, a través del portal de transparencia del gobierno autonómico (http://www.gobiernodecanarias.org/transparencia/).

\section{Su potencial en el análisis de la dimensión espacial y temporal de los sistemas agrarios}

Partiendo de lo ya expuesto anteriormente, el diseño e implementación del Mapa de Cultivos de Canarias tiene una perspectiva eminentemente espacial. La identificación, delimitación y caracterización de cada unidad de explotación ha dado lugar a diferentes bases gráficas en formato vectorial, por campaña e isla, que permiten una medición sistemática de la localización, distribución, asociación e interrelación entre ellas. De este modo, se facilita una lectura del sistema agrario contrastada con la realidad territorial, no exenta de las limitaciones impuestas por la complejidad, heterogeneidad y dinamismo de aquello que se mide, sus periodos de actualización y las particularidades del modelo informático que lo abstrae y codifica.

Cubierto el análisis espacial, recientemente se incorporaría la dimensión temporal (no contemplada en su origen), con el planteamiento de un método que cruzase, corrigiese y adaptase los datos disponibles de diferentes campañas para medir su evolución considerando las variables temporal y espacial de forma combinada. Este ejercicio no estaría exento de problemas, dado que cada base gráfica constituye una foto fija del sistema agrario en el momento en el que se procedió a la toma de datos en campo, realizada con una metodología, fuentes y criterios que han evolucionado con el proceso de aprendizaje, no considerando inicialmente la posibilidad de contemplar la variable temporal unida a la espacial. Por este motivo, la suma de la complejidad inherente a aquello que se quiere medir, los cambios en la forma de medirlo, las fuentes utilizadas como base, el momento en el que se llevó a cabo la observación, el contexto en el que se ha desarrollado, entre otros muchos factores, condicionaron el ajuste entre bases gráficas, requiriendo de cierta precaución en su procesamiento e interpretación.

A modo de ejemplo, las fuentes cartográficas de base han ido adquiriendo una mayor precisión y nivel de detalle en cada campaña. De este modo, en la última disponible para cada isla suelen detectarse edificaciones, depósitos, caminos, entre otros elementos no identificados en su antecesora, debiendo descartarlos como superficie cultivada en ambas, excepto en los casos en los que se trate de cambios reales (reconocibles mediante fotointerpretación). Del mismo modo, esta precisión en la delimitación lleva a un mejor ajuste de los perímetros de cada recinto, descartando 
García Cruz, J.I.; Febles Ramírez, M.F.; Pestana Pérez, G. (2017): “Las Tecnologías de la Información Geográfica aplicadas a la sistematización de los sistemas agrarios. Bases para el estudio de su dimensión espacial y temporal. El caso del mapa de cultivos de canarias (España)", GeoFocus (Artículos), no 19, p. 181-206. ISSN: 1578-5157 http://dx.doi.org/10.21138/GF.558

superficie que realmente no estuviera cultivada, así como reconociendo aquella que hubiera sido inicialmente descartada, como es el caso de la localizada en los perímetros urbanos. Desde el punto de vista de los criterios de interpretación, con el incremento de la precisión de la cartografía base y las capacidades de las herramientas destinadas a la recopilación de datos en campo, la posibilidad de delimitar con mayor precisión cada tipo de cultivo ha reducido el número de recintos reconocido con algún tipo de asociación. De este modo, se hace necesario matizar algunos cambios de cultivos, ya que una parte de éstos no debieran interpretarse como tal, aspecto condicionado por la complejidad de los rotacionales, entre otros aspectos. En definitiva, sobre la base del último mapa disponible de cada isla, se desarrolla un método de cruce, reconocimiento y corrección de posibles desajustes e incoherencias con su antecesor, obteniendo posteriormente una aproximación al dinamismo del sistema agrario, a través de la medición de las entradas, salidas, cambios o permanencias en el sistema, no exenta de ciertas limitaciones (ver Febles y García 2015a).

De este modo, la lectura del Mapa de Cultivos puede dividirse en dos bloques interrelacionados. Por un lado, la lectura de cada foto fija (campaña), que permite conocer la entidad territorial, distribución y características del sistema agrario y subsistema cultivo, mientras que, por otro, la consideración de un mapa de entrada (primera campaña realizada en la isla) y uno de salida (última campaña), facilita medir la evolución experimentada mediante su cruce y comparación, aplicando de forma intermedia un proceso de ajuste y corrección de las incoherencias derivadas de posibles diferencias entre las fuentes, métodos y criterios empleados en cada una de ellas.

Dado que el Mapa de Cultivos de cada isla cubre y clasifica la totalidad de la superficie insular, debemos establecer una primera distinción entre las clases asimilables dentro del sistema agrario del resto de superficies. Así, por sistema agrario se ha entendido la suma de la superficie cultivada, la considerada sin cultivo y el pastizal (Febles y García, 2015a). En concreto, por superficie cultivada se entiende todos aquellos recintos (unidades de producción) en los que se reconoce un uso agrícola activo (identificación de alguna tipología), el pastizal hace referencia a la presencia de pastos con un uso agropecuario, así como aquellas superficies anteriormente cultivas que actualmente no presentan un uso activo (pero sostienen un potencial de incorporación) se identifican como sin cultivo (asimilable con el concepto abandono). De este modo, se establece una distinción entre el sistema agrario, englobando cultivo, pastizal y sin cultivo, y el subsistema cultivo, centrado únicamente en la superficie cultivada dentro de éste.

Por el contrario, fuera de este sistema se engloban los eriales y el monte. En concreto, por erial se entiende toda aquella superficie bajo unas características que dificulta, impiden o precisan de un elevado esfuerzo e inversión para su puesta en producción, agrupando a una amplia diversidad de usos (áreas urbanas, coladas volcánicas, playas, zonas de topografía abrupta y elevada pendiente, abandonos de largo plazo, etc.), mientras que el monte agrupa las áreas ocupadas por diversas formaciones vegetales. Para ambos casos, se trata de reconocimientos y delimitaciones menos precisos que en el sistema agrario, dado que éstos usos quedan fuera del interés de la fuente. De este modo, ante la necesidad de disponer de datos sobre el monte o los eriales de forma más precisa y detallada, se recomienda el uso de otras fuentes oficiales, como el mapa forestal, montes de utilidad pública u ocupación del suelo, entre otros. 
García Cruz, J.I.; Febles Ramírez, M.F.; Pestana Pérez, G. (2017): “Las Tecnologías de la Información Geográfica aplicadas a la sistematización de los sistemas agrarios. Bases para el estudio de su dimensión espacial y temporal. El caso del mapa de cultivos de canarias (España)", GeoFocus (Artículos), $n^{\circ}$ 19, p. 181-206. ISSN: 1578-5157 http://dx.doi.org/10.21138/GF.558

Como resultado, la distinción de la superficie asimilable como sistema agrario permite localizar, medir y caracterizar cada sistema agrario insular (ver Figura 5), a la vez que facilita la comprobación de su dinámica interna (cambios de superficie entre el cultivo, sin cultivo y pastizal) y externa (entradas y salidas en el sistema) (ver Tabla 3 y 4), con el añadido de que esta medición siempre lleva asociadas las variables espacial y temporal que permiten ubicar el proceso y su evolución en el mapa (ver Figura 7).

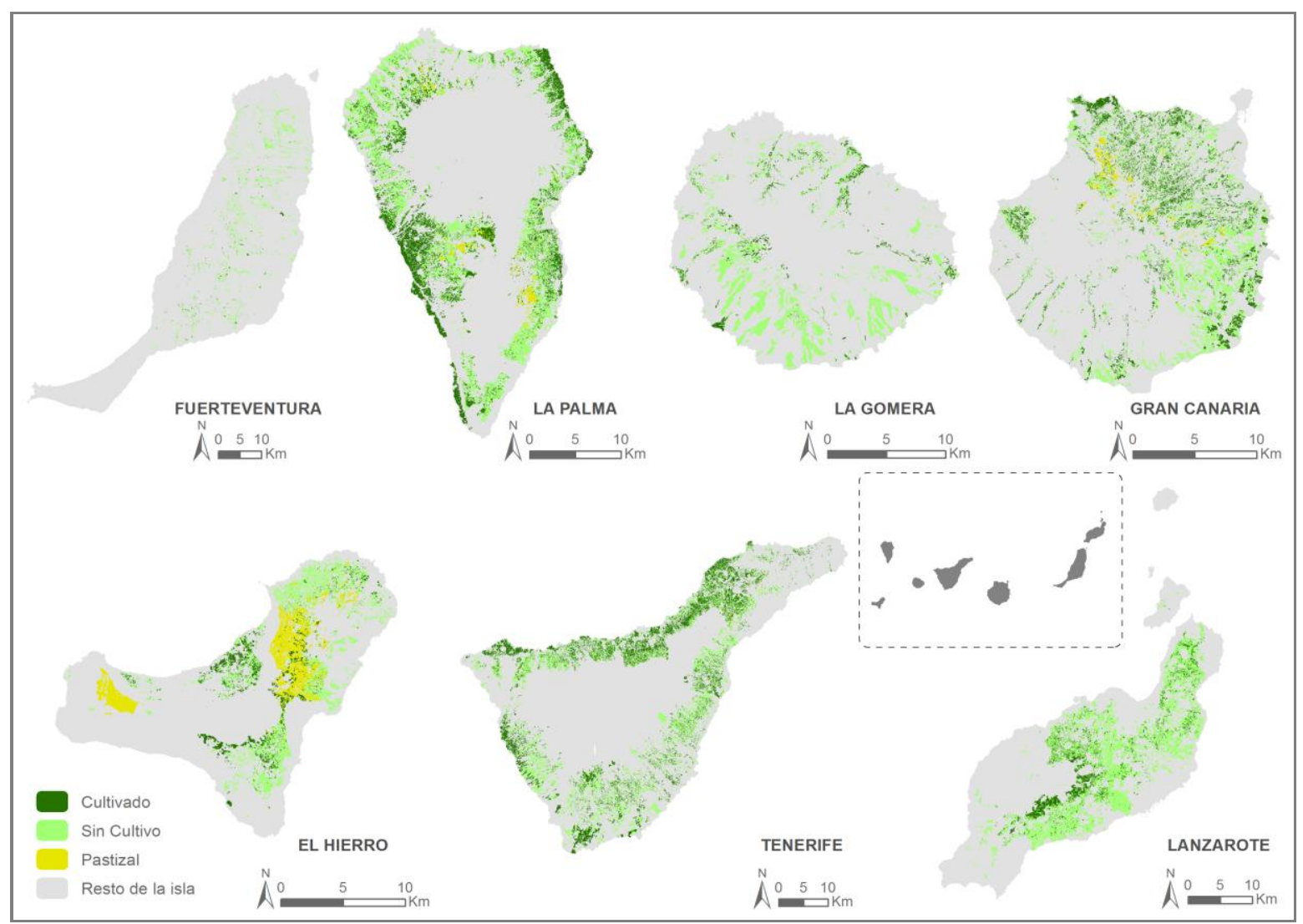

Figura 5. Caracterización de los sistemas agrarios insulares de Canarias.

Fuente: Mapa de cultivos de Canarias. Elaboración propia 
García Cruz, J.I.; Febles Ramírez, M.F.; Pestana Pérez, G. (2017): "Las Tecnologías de la Información Geográfica aplicadas a la sistematización de los sistemas agrarios. Bases para el estudio de su dimensión espacial y temporal. El caso del mapa de cultivos de canarias (España)", GeoFocus (Artículos), no 19, p. 181-206. ISSN: 1578-5157 http://dx.doi.org/10.21138/GF.558

Tabla 3. Resumen de la evolución de superficies en los sistemas agrarios insulares

\begin{tabular}{|l|r|r|r|r|r|r|r|r|}
\hline \multirow{2}{*}{ ISLA } & \multicolumn{2}{|c|}{ Sup. Cultivada } & \multicolumn{2}{c|}{ Sup. Sin cultivo } & \multicolumn{2}{c|}{ Sup. Pastizal } & \multicolumn{2}{c|}{ Sup. Sistema agrario } \\
\cline { 2 - 9 } & Entrada & \multicolumn{1}{c|}{ Salida } & Entrada & \multicolumn{1}{c|}{ Salida } & Entrada & \multicolumn{1}{c|}{ Salida } & Entrada & \multicolumn{1}{c|}{ Salida } \\
\hline El Hierro & $1.149,9$ & $1.013,6$ & $3.913,9$ & $2.948,5$ & 769,1 & $2.078,1$ & $5.832,9$ & $6.040,2$ \\
\hline La Palma & $6.269,0$ & $6.612,4$ & $11.256,3$ & $10.989,3$ & 816,9 & 710,7 & $18.342,2$ & $18.312,6$ \\
\hline La Gomera & 919,6 & 712,3 & $3.981,2$ & $4.115,8$ & 0,2 & 0 & $4.901,1$ & $4.828,1$ \\
\hline Tenerife & $19.976,9$ & $18.368,0$ & $22.651,2$ & $24.572,8$ & 283,8 & 179,2 & $42.911,9$ & $43.119,9$ \\
\hline Gran Canaria & $12.389,4$ & $10.371,8$ & $19.343,4$ & $20.661,6$ & $1.343,3$ & $1.512,7$ & $33.076,2$ & $32.546,1$ \\
\hline Fuerteventura & 541,7 & 515,3 & $8.392,1$ & $8.573,7$ & 0 & 0,7 & $8.933,9$ & $9.089,4$ \\
\hline Lanzarote & $4.143,2$ & $3.819,2$ & $18.551,3$ & $18.902,2$ & 0 & 3,8 & $22.694,6$ & $22.725,2$ \\
\hline Canarias & $45.389,9$ & $41.412,7$ & $88.089,5$ & $90.763,6$ & $3.213,3$ & $4.485,2$ & $136.692,7$ & $136.661,6$ \\
\hline
\end{tabular}

Unidades de superficie (Sup.): hectáreas. Campañas de entrada (El Hierro 2001-2002, La Palma 2001-2003, La Gomera 2002-2003, Tenerife 1998-2001, Gran Canaria 2000-2002, Fuerteventura 2002-2004, Lanzarote 2002-2003), campañas de salida (El Hierro 2014-2015, La Palma 2008-2009, La Gomera 2009-2010, Tenerife 2007-2008, Gran Canaria 2012-2013, Fuerteventura 2013-2014, Lanzarote 2014).

Fuente: Mapa de cultivos de Canarias. Elaboración propia.

Tabla 4. Resumen de la dinámica de los sistemas agrarios en cada contexto insular

\begin{tabular}{|l|r|r|r|r|r|r|r|r|r|r|}
\hline \multirow{1}{*}{ ISLA } & \multicolumn{2}{|c|}{$\begin{array}{c}\text { Entradas en } \\
\text { el sistema }\end{array}$} & \multicolumn{2}{c|}{$\begin{array}{c}\text { Salidas del } \\
\text { sistema }\end{array}$} & \multicolumn{2}{c|}{$\begin{array}{c}\text { Permanencia en } \\
\text { el sistema }\end{array}$} & \multicolumn{2}{c|}{$\begin{array}{c}\text { Cambios } \\
\text { internos en el } \\
\text { sistema }\end{array}$} & \multicolumn{2}{c|}{$\begin{array}{c}\text { Permanencia } \\
\text { fuera del } \\
\text { sistema }\end{array}$} \\
\cline { 2 - 12 } & \multicolumn{1}{|r|}{ Sup. } & $\%$ & \multicolumn{1}{c|}{ Sup. } & $\%$ & \multicolumn{1}{c|}{ Sup. } & \multicolumn{1}{c|}{$\%$} & \multicolumn{1}{c|}{ Sup. } & $\%$ & \multicolumn{1}{c|}{ Sup. } & $\%$ \\
\hline El Hierro & 637,7 & 2,4 & 430,4 & 1,6 & $3.356,4$ & 12,5 & $2.046,2$ & 7,6 & $20.313,9$ & 75,8 \\
\hline La Palma & 712,6 & 1 & 742,3 & 1,1 & $14.172,6$ & 20,1 & $3.427,3$ & 4,9 & $51.541,5$ & 73,0 \\
\hline La Gomera & 85,0 & 0,2 & 157,9 & 0,4 & $4.269,3$ & 11,6 & 473,8 & 1,3 & $31.761,2$ & 86,4 \\
\hline Tenerife & $2.004,2$ & 1 & 1796,0 & 0,9 & $30.804,5$ & 15,2 & $10.311,3$ & 5,1 & $158.152,9$ & 77,9 \\
\hline Gran Canaria & $2.092,4$ & 1,3 & $2.622,5$ & 1,7 & $23.215,9$ & 14,9 & $7.237,8$ & 4,6 & $120.504,6$ & 77,4 \\
\hline Fuerteventura & 236,0 & 0,1 & 80,5 & 0,1 & $8.287,2$ & 5,0 & 566,2 & 0,3 & $156.192,5$ & 94,5 \\
\hline Lanzarote & 262,0 & 0,3 & 231,4 & 0,3 & $19.504,8$ & 23,1 & $2.958,4$ & 3,5 & $61.324,3$ & 72,8 \\
\hline Canarias & $6.029,9$ & 0,8 & $6.061,0$ & 0,8 & $103.610,6$ & 13,9 & $27.021,1$ & 3,6 & $599.791,0$ & 80,8 \\
\hline
\end{tabular}

Unidades de superficie (Sup.): hectáreas. Campañas de entrada (El Hierro 2001-2002, La Palma 2001-2003, La Gomera 2002-2003, Tenerife 1998-2001, Gran Canaria 2000-2002, Fuerteventura 2002-2004, Lanzarote 2002-2003), campañas de salida (El Hierro 2014-2015, La Palma 2008-2009, La Gomera 2009-2010, Tenerife 2007-2008, Gran Canaria 2012-2013, Fuerteventura 2013-2014, Lanzarote 2014).

Fuente: Mapa de cultivos de Canarias. Elaboración propia.

Del mismo modo, puede obtenerse una lectura espacial y temporal del subsistema cultivo, entrando en los pormenores sobre la categoría, agrupación y tipo de cultivo, las técnicas empleadas en cada recinto (invernadero, jable, enarenado, gavias) o la presencia del regadío, sosteniendo igualmente su dimensión espacial y temporal, a través de su representación en el mapa. Así, la variable espacial permite identificar, no sólo la superficie ocupada, sino también una lectura de su distribución en cada espacio insular, ya sea considerando sus límites administrativos (municipios) o altitudinales (pisos o franjas), entre otros, a lo que se añade la visión temporal, con la posibilidad de comparar los datos entre campañas (ver Tabla 5 y Figura 6) o una combinación de ambos 
García Cruz, J.I.; Febles Ramírez, M.F.; Pestana Pérez, G. (2017): “Las Tecnologías de la Información Geográfica aplicadas a la sistematización de los sistemas agrarios. Bases para el estudio de su dimensión espacial y temporal. El caso del mapa de cultivos de canarias (España)", GeoFocus (Artículos), no 19, p. 181-206. ISSN: 1578-5157 http://dx.doi.org/10.21138/GF.558

localizando los lugares donde acontecen las entradas, salidas, permanencias o cambios en el sistema (ver Figura 7).

Tabla 5. Resumen del balance de superficies de los subsistemas cultivo insulares desagregado por bloques de cultivo

\begin{tabular}{|l|r|r|r|r|r|r|r|r|}
\hline \multicolumn{1}{|c|}{ ISLA } & \multicolumn{1}{c|}{ Huerta } & Tomate & Frutales & Platanera & \multicolumn{1}{c|}{ Otros } & \multicolumn{1}{c|}{ Viña } & Ornamentales & Balance insular \\
\hline El Hierro & $-16,6$ & 0,01 & 316,0 & 0,3 & $-172,4$ & $-262,1$ & $-1,4$ & $-136,3$ \\
\hline La Palma & 337,8 & $-2,7$ & 274,0 & $-268,8$ & 91,6 & $-96,4$ & 7,9 & 343,4 \\
\hline La Gomera & $-64,1$ & $-0,3$ & 0,3 & $-62,9$ & $-9,9$ & $-68,9$ & 1,5 & $-204,4$ \\
\hline Tenerife & $-2.086,3$ & $-557,9$ & 419,3 & $-107,3$ & 606,5 & 110,8 & 6,0 & $-1.608,9$ \\
\hline Gran Canaria & $-665,6$ & $-1.517,2$ & 345,9 & $-267,9$ & 116,3 & 31,5 & $-60,5$ & $-2.017,6$ \\
\hline Fuerteventura & 92,2 & $-28,4$ & 108,4 & $-13,6$ & $-195,7$ & 3,5 & 7,2 & $-26,5$ \\
\hline Lanzarote & 444,9 & 1,1 & 29,9 & 0,3 & -438.8 & $-364,1$ & 2,6 & $-324,1$ \\
\hline Canarias & $-1.957,8$ & $-2.105,4$ & $1.493,9$ & $-719,9$ & $-2,4$ & $-648,7$ & $-36,7$ & $-3.977,2$ \\
\hline
\end{tabular}

Unidades de superficie: hectáreas. Campañas de entrada (El Hierro 2001-2002, La Palma 2001-2003, La Gomera 20022003, Tenerife 1998-2001, Gran Canaria 2000-2002, Fuerteventura 2002-2004, Lanzarote 2002-2003), campañas de salida (El Hierro 2014-2015, La Palma 2008-2009, La Gomera 2009-2010, Tenerife 2007-2008, Gran Canaria 2012-2013, Fuerteventura 2013-2014, Lanzarote 2014).

Fuente: Mapa de cultivos de Canarias. Elaboración propia. 
García Cruz, J.I.; Febles Ramírez, M.F.; Pestana Pérez, G. (2017): "Las Tecnologías de la Información Geográfica aplicadas a la sistematización de los sistemas agrarios. Bases para el estudio de su dimensión espacial y temporal. El caso del mapa de cultivos de canarias (España)", GeoFocus (Artículos), $n^{\circ}$ 19, p. 181-206. ISSN: 1578-5157 http://dx.doi.org/10.21138/GF.558

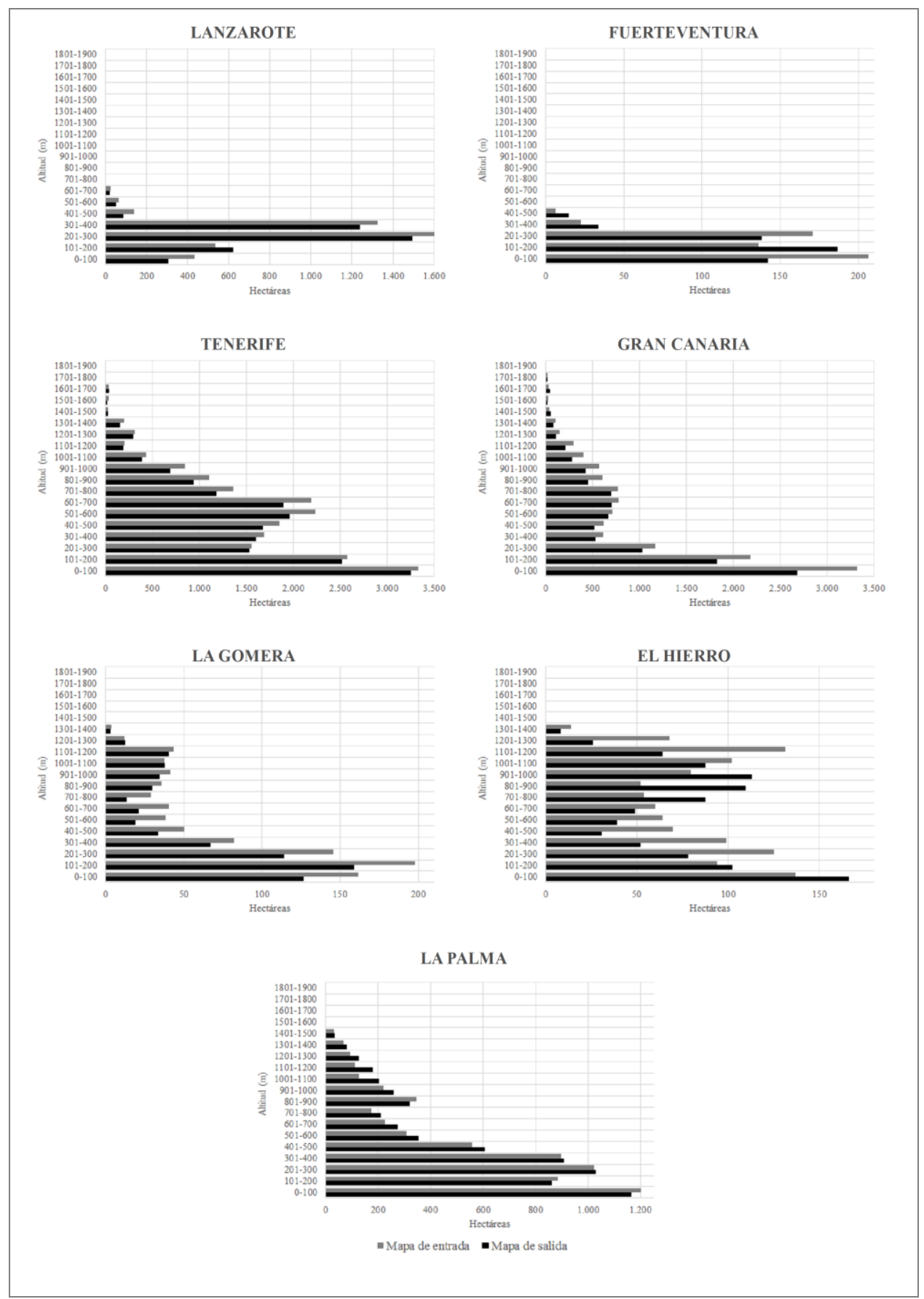

Unidades: hectáreas. Campañas de entrada (El Hierro 2001-2002, La Palma 2001-2003, La Gomera 2002-2003, Tenerife 1998-2001, Gran Canaria 2000-2002, Fuerteventura 2002-2004, Lanzarote 2002-2003), campañas de salida (El Hierro 2014-2015, La Palma 2008-2009, La Gomera 2009-2010, Tenerife 2007-2008, Gran Canaria 2012-2013, Fuerteventura 2013-2014, Lanzarote 2014).

Figura 6. Evolución comparada de la distribución altitudinal de los subsistemas cultivo insulares. 
García Cruz, J.I.; Febles Ramírez, M.F.; Pestana Pérez, G. (2017): “Las Tecnologías de la Información Geográfica aplicadas a la sistematización de los sistemas agrarios. Bases para el estudio de su dimensión espacial y temporal. El caso del mapa de cultivos de canarias (España)", GeoFocus (Artículos), $n^{\circ}$ 19, p. 181-206. ISSN: 1578-5157 http://dx.doi.org/10.21138/GF.558

Fuente: Mapa de cultivos de Canarias. Elaboración propia

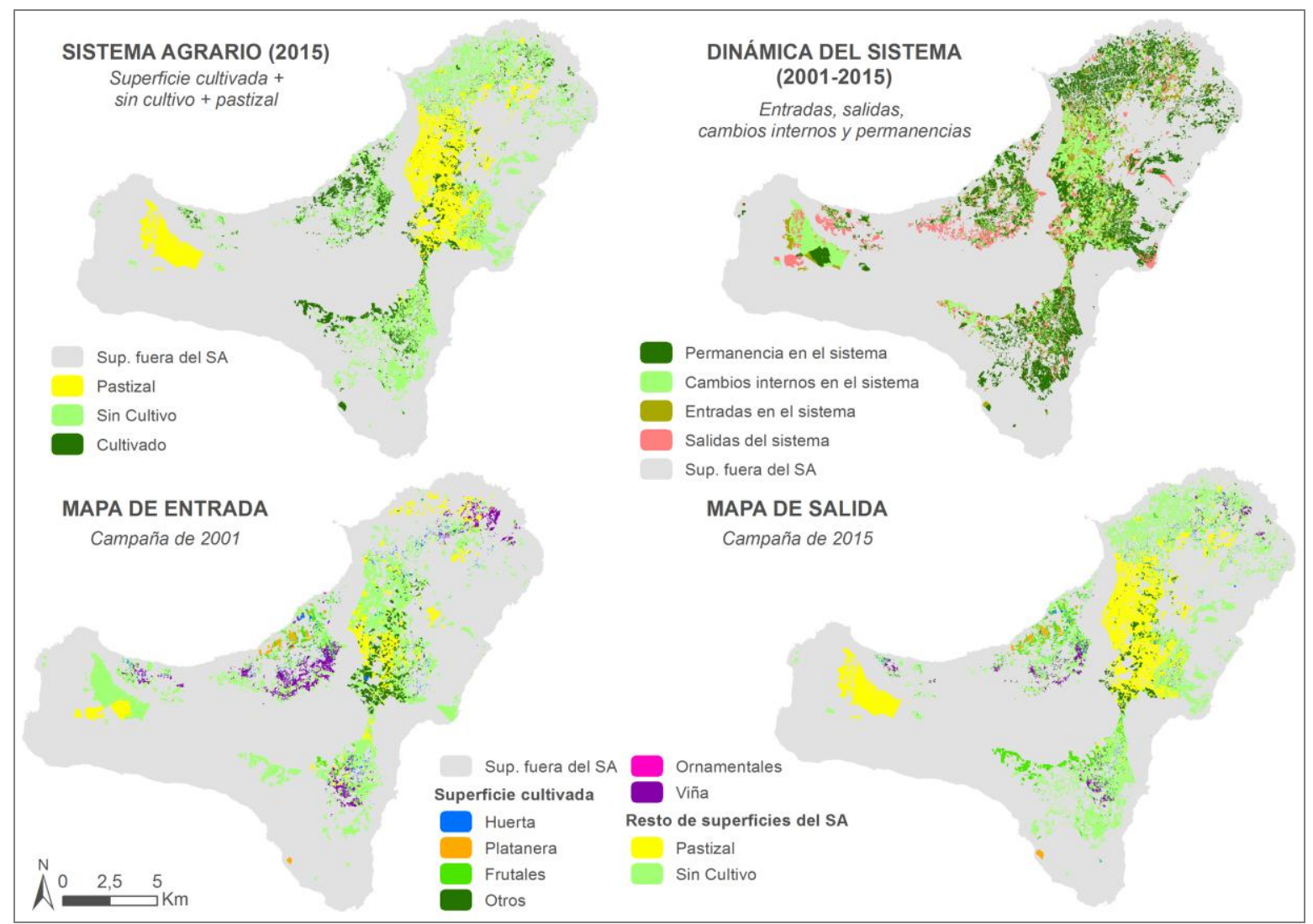

Figura 7. Ejemplo de las diferentes lecturas que puede realizarse del sistema agrario y subsistema cultivo (El Hierro).

Fuente: Mapa de cultivos de Canarias. Elaboración propia.

Con todo ello, el Mapa de Cultivos ha servido de base para corroborar, junto con otras fuentes, la dinámica experimentada por los sistemas agrarios insulares en tiempos recientes. Así, son evidentes las particularidades de cada ámbito insular en cuanto a su relieve, fachadas de sotavento y barlovento, pisos bioclimáticos (ver Martín, 2000 y García Rodríguez, 2013), pudiendo realizar una comprobación cuantitativa de sus principales tendencias (ver Godenau y Nuez, 2015). De este modo, los datos corroboran una concentración de la superficie cultivada entre la medianía y el litoral, coincidiendo con los ejes de desarrollo insulares surgidos a partir del cambio de modelo socio-económico experimentado desde mediados del siglo XX, entre otros aspectos (Simancas, 2007; Hernández, Simancas y García, 2010; García y Simancas, 2011; García Rodríguez, 2013; Godenau y Nuez, 2015).

Por otro lado, al trabajar sobre bases gráficas implementadas a través de un SIG, éstas pueden interrelacionarse con aquellas derivadas de otras administraciones, empresas, instituciones, centros de investigación, etc., facilitando una lectura aún más completa de la realidad territorial abstraída en las mismas. Un ejemplo de lo anteriormente descrito ha sido la establecida entre la 
García Cruz, J.I.; Febles Ramírez, M.F.; Pestana Pérez, G. (2017): “Las Tecnologías de la Información Geográfica aplicadas a la sistematización de los sistemas agrarios. Bases para el estudio de su dimensión espacial y temporal. El caso del mapa de cultivos de canarias (España)", GeoFocus (Artículos), no 19, p. 181-206. ISSN: 1578-5157 http://dx.doi.org/10.21138/GF.558

delimitación del suelo urbanizado en Canarias (ver García Cruz, 2013) y la dinámica experimentada recientemente en los sistemas agrarios insulares.

Su combinación ha permitido evidenciar un importante cambio de tendencia, en el que la superficie urbanizada en las islas $(6 \%)$ ha superado a la cultivada $(5,58 \%)$. Aun así, su relación espacial, sin entrar en otras relaciones causales, ha permitido descartar la acción de la presión urbanizadora sobre el espacio agrícola como principal agente modificador del territorio, evidenciándose, en su defecto, la perdida de superficie cultivada por su tránsito hacia el abandono, mientras la urbanización ha ocupado preferentemente espacios identificados como erial o abandonos prolongados con anterioridad a su clasificación como suelo urbanizable (García Cruz, 2013; Febles y García, 2015b).

Como resultado, las bases gráficas vectoriales sobre las que se desarrolla el Mapa de Cultivos de Canarias, permiten el desarrollo de un análisis espacial de los sistemas agrarios y subsistemas cultivo, a los que se les permite añadir la variable temporal, facilitando la identificación, localización, delimitación y caracterización de sus dinámicas (entradas, salidas o cambios internos) (ver Figura 7). Del mismo modo, la interoperabilidad inherente a estas bases gráficas abre todo un abanico de posibilidades de interacción con las provenientes de otras fuentes, desarrollando una lectura más amplia del territorio dentro del marco de las TIG (tecnológico, conceptual y metodológico $)^{4}$, así como comparar su lectura con las realizadas anteriormente sobre otras fuentes.

Un ejemplo de lo anterior puede ser la evolución experimentada por los cultivos de exportación que ocupan parcialmente los litorales insulares. Considerando esta franja altitudinal (entre los 0 y 100 metros de altitud), en ella se aprecia el desarrollo de la platanera, con un 59,9\% de la superficie y, en menor medida, por la huerta $(17,76 \%)$. Por el contrario, se identifica una importante disminución del peso de otro cultivo de exportación, como es el tomate, pasando del $21 \%$, al $8,8 \%$ actual. Se trata de una reducción de 2.105,44 ha en un periodo aproximado de 10 años, que deja a este cultivo en las $1.468,78$ ha actuales, descenso muy marcado al compararlo con las cifras publicadas en décadas anteriores, donde éste cultivo alcanzó las 4.513 ha estimadas sólo en Tenerife y Gran Canaria en 1951 (Villalva, 1978).

En resumidas cuentas, si consideramos únicamente las capacidades del Mapa de Cultivos de Canarias de forma aislada, éste nos permite localizar y dimensionar el sistema agrario, pudiendo diferenciar cada una de sus partes (superficie cultivada, sin cultivo y pastizal), a la vez que conocer los pormenores de la superficie cultivada (agrupación y tipo de cultivo, técnica, etc.), hasta la escala de unidad de producción (recinto). Todo ello sin perder una dimensión espacial y temporal que permite identificar los efectos de diferentes procesos (ej. aparición de nuevos cultivos, desaparición hacia el abandono, urbanización de superficies anteriormente cultivadas, etc.), pudiendo localizar y medir sus efectos en el territorio.

\footnotetext{
${ }^{4}$ En este sentido, pueden tomarse como ejemplo algunos trabajos como los de García y Simancas (2011) sobre el estudio del paisaje en las áreas protegidas de Canarias o García Cruz (2013) sobre el impacto territorial del tercer boom turístico-inmobiliario en Canarias, usando ambas como una de sus fuentes territoriales de base el Mapa de Cultivos.
} 
García Cruz, J.I.; Febles Ramírez, M.F.; Pestana Pérez, G. (2017): "Las Tecnologías de la Información Geográfica aplicadas a la sistematización de los sistemas agrarios. Bases para el estudio de su dimensión espacial y temporal. El caso del mapa de cultivos de canarias (España)", GeoFocus (Artículos), $n^{\circ}$ 19, p. 181-206. ISSN: 1578-5157 http://dx.doi.org/10.21138/GF.558

Si a esto le unimos la posibilidad de que esta base gráfica sea utilizada por un número de usuarios más amplio, así como interaccionar con otras bases gráficas, yendo más allá de su uso original, se amplía su aplicabilidad, pudiendo destinarse a la ordenación del territorio o el estudio del paisaje, entre otras temáticas, añadiendo una visión que puede enriquecer la lectura que pretenda realizarse de las formas, procesos y estructuras territoriales.

\section{Conclusiones. El potencial de una lectura que interrelaciona la dimensión espacial y temporal del sistema agrario}

Surgido de la necesidad de desarrollar una fuente adaptada a las particularidades de los sistemas agrarios insulares, el Mapa de Cultivos de Canarias se ha posicionado como una fuente básica para su comprensión y posterior diseño de políticas de intervención.

Lejos de haber logrado todos sus objetivos, el abstraer una realidad compleja, heterogénea y dinámica, sostiene un continuo proceso de aprendizaje, donde la implementación de cambios y mejoras tratan de adaptar la fuente a unas necesidades de información y conocimiento cada vez más amplias y diversas.

Esto ha supuesto que la fuente, lejos de sus pretensiones originales, centradas en las necesidades propias de la administración que la desarrolla, comience a enfocarse hacia otras temáticas, como la ordenación territorial, el estudio del paisaje, la enseñanza y difusión de las particularidades de la agricultura en el archipiélago, entre otros muchos, lo que justifica y demanda el ingente trabajo que supone a la administración pública Canaria (presupuestario, material y personal) la planificación, desarrollo y gestión de esta fuente, siempre y cuando sepa responder a estas demandas.

Su implementación, con el apoyo de TIG, ha permitido agilizar su construcción, a través de sucesivas campañas de toma de datos y su gestión posterior, facilitando una visión espacial de un fenómeno con claras implicaciones territoriales, permitiendo conocer, no sólo la entidad territorial de sus principales cultivos, sino además situar las dinámicas, relaciones y asociaciones donde tienen lugar, con una precisión a escala 1:2.500. A esto se le ha sumado últimamente la visión temporal, en la que la evolución reciente de los sistemas agrarios insulares puede medirse unida a la dimensión espacial del fenómeno.

Por este motivo, la fuente facilita la localización y cuantificación de los procesos que se producen en los sistemas agrarios insulares de Canarias, con una precisión y nivel de detalle superior a las disponibles a escala estatal, posicionándose como una fuente relevante para conocer y actuar sobre estos espacios, sin que esto descarte la posibilidad de combinarlas.

De su explotación es posible extraer mediciones sistemáticas que permiten realizar una lectura de su localización, dimensión, distribución y evolución reciente, pudiendo distinguir sus dinámicas internas y externas (entradas, salidas, cambios o permanencias en el sistema agrario y subsistema cultivo). 
García Cruz, J.I.; Febles Ramírez, M.F.; Pestana Pérez, G. (2017): “Las Tecnologías de la Información Geográfica aplicadas a la sistematización de los sistemas agrarios. Bases para el estudio de su dimensión espacial y temporal. El caso del mapa de cultivos de canarias (España)", GeoFocus (Artículos), no 19, p. 181-206. ISSN: 1578-5157 http://dx.doi.org/10.21138/GF.558

En definitiva, se trata de cuestiones con un reflejo territorial captable y medible que permite su análisis espacial. Por este motivo, la utilización de TIG en la confección de bases gráficas, facilita una visión combinada de la dimensión espacial y temporal que amplía la lectura que puede realizarse del territorio, siempre y cuando sean capaces de captar de forma adecuada las particularidades de los sistemas agrarios.

La existencia de esta fuente de información y su potencial combinación con otras capas de información territoriales y sociodemográficas permitirá un diagnóstico más rico e integrado de la realidad de los territorios identificados como rurales (Godenau, Hernández y Febles, 2004).

\section{Referencias bibliográficas}

Aguilera Ardilla, M.J.; Borderías Uribeondo, M.P.; González Yanci, M.P. y Santos Preciado, J.M. (2012): Geografía general II: geografía humana. Madrid, UNED (col. Unidades Didácticas).

Alonso Sanz, R. (1990): “Estadística y agricultura”, Estadística Española, 32, 125, pp. 477-503.

Bosque Sendra, J. (1992): Sistemas de información geográfica. Madrid Ediciones Rialp.

Bosque Sendra, J. (1999): "La ciencia de la información geográfica y la geografía", Actas VII Encuentro de Geógrafos de América Latina, San Juan de Puerto Rico.

Buzai, G.D. (2010): “Análisis espacial con sistemas de información geográfica: sus cinco conceptos fundamentales", en Buzai, G.D. (Ed.): Geografía y sistemas de información geográfica. Aspectos conceptuales y aplicaciones. Luján, Universidad de Luján, pp. 163-195.

Buzai, G.D., y Baxendale, C. A. (2006): Análisis socioespacial con sistemas de información geográfica. Buenos Aires, Lugar Editorial.

Buzai, G.D., Baxendale, C.A., y Cruz, M.R. (2009): "Fases de un proyecto de investigación en estudios de geografía aplicada basados en el uso de sistemas de información geográfica", Fronteras, 8, pp. 31-40.

Capel Sáez, H. (2005): Las TIG en los concursos de habilitación para profesores titulares de geografía humana. Una cuestión nada anecdótica. Barcelona, Biblio 3W. Revista Bibliográfica de Geografía y Ciencias Sociales. [Consulta: 19-12-2016]. Disponible en http://www.ub.edu/geocrit/b3w-620.htm

Capel Sáez, H. (2009): Geografía en la red a comienzos del tercer milenio. Por una ciencia solidaria y en colaboración. Barcelona, Biblio 3W. Revista Bibliográfica de Geografía y Ciencias Sociales. [Consulta: 19-12-2016]. Disponible en http://www.ub.edu/geocrit/sn/sn-313.htm

Dobson, J. E. (1983): “Automated Geography”, The Professional Geographer, 2, 35, pp. 135-143.

Dobson, J. E. (1993): "The Geographic Revolution: A Retrospective on the Age of Automated Geography”, The Professional Geographer, 4, 45, pp. 431-439.

Egea Ibáñez, J. (2005): Sistema agrario: proyecto evolutivo estable. Madrid, Editorial MundiPrensa.

Évora Brondo, J.A. (2015): "Recorrido por las fuentes de información cartográfica", en Pestana Pérez, G.; Febles Ramírez, M. F. y de la Rosa Villar, B. (coord.): La agricultura Canaria a 
García Cruz, J.I.; Febles Ramírez, M.F.; Pestana Pérez, G. (2017): “Las Tecnologías de la Información Geográfica aplicadas a la sistematización de los sistemas agrarios. Bases para el estudio de su dimensión espacial y temporal. El caso del mapa de cultivos de canarias (España)", GeoFocus (Artículos), $n^{\circ}$ 19, p. 181-206. ISSN: 1578-5157 http://dx.doi.org/10.21138/GF.558

principios del siglo XXI. Análisis de los mapas de cultivos de Canarias. Madrid, Ministerio de Agricultura, Alimentación y Medio Ambiente, pp. 23-41.

Febles Ramírez, M.F.; García Cruz, J.I. (2015a): "Explotación y presentación de la información del mapa de cultivos de canarias", en Pestana Pérez, G.; Febles Ramírez, M. F. y de la Rosa Villar, B. (coord.): La agricultura Canaria a principios del siglo XXI. Análisis de los mapas de cultivos de Canarias. Madrid, Ministerio de Agricultura, Alimentación y Medio Ambiente, pp. 67-80.

Febles Ramírez, M.F.; García Cruz, J.I. (2015b): "Canarias”, en Pestana Pérez, G.; Febles Ramírez, M. F. y de la Rosa Villar, B. (coord.): La agricultura Canaria a principios del siglo XXI. Análisis de los mapas de cultivos de Canarias. Madrid, Ministerio de Agricultura, Alimentación y Medio Ambiente, pp. 383-402.

Fernández-Palacios, J. M.; Arévalo Sierra, J. R. y Rüdiger, O. (2004): Canarias. Ecología, medio ambiente y desarrollo. San Cristóbal de La Laguna, Centro de la Cultura Popular Canaria.

García Cruz, J.I. (2013): El impacto territorial del tercer boom turístico de Canarias. San Cristóbal de La Laguna, Servicio de Publicaciones de la Universidad de La Laguna.

García Cruz, J.I. (2015): "El análisis del impacto territorial del tercer boom turístico en Canarias (España) a través de la aplicación de un sistema de información geográfica (SIG)", Cuadernos de turismo, 36, pp. 219-245.

García Cruz, J.I. y Simancas Cruz, M.R. (2011): "El paisaje de las áreas protegidas de Canarias", en Simancas Cruz, M.R. y Cortina Ramos, A (coord.): Retos y perspectivas de la gestión del Paisaje de Canarias. Reflexiones en relación con el $10^{\circ}$ aniversario de la firma del Convenio Europeo del Paisaje. Santa Cruz de Tenerife, Observatorio del Paisaje de Canarias. Universidad Internacional Menéndez Pelayo, pp. 251-269.

García Rodríguez, J.L. (2013): "Paisajes agrarios de Canarias", Anales de Geografía, 33, 1, pp. 93132.

García Rodríguez, J.L. (2015): "La agricultura mínima. La cuantificación de la superficie cultivada en Canarias", en de La Riva, J.; Ibarra, P.; Montorino. R.; Rodríguez, M. (Ed.): Análisis espacial y representación geográfica: innovación y aplicación. Zaragoza, Universidad de Zaragoza-AGE, pp. 2015-2034.

Godenau, D.; Hernández López, M.; Febles Ramírez, M. (2004): “Tipificación de zonas rurales a través del análisis multivariante de información extraída de sistemas de información geográfica", Revista Española de Estudios Agrosociales y Pesqueros, 203, pp. 85-109

Godenau, D. y Nuez Yánez, J.S. (2015): "El sector agrario”, en Padrón Marrero, D. y Rodríguez Martín, J.A. (coord.): Economía de Canarias. Dinámica, estructura y retos. Valencia, Tirant Lo Blanch, pp. 389-421.

Gómez Delgado, M., y Barredo Cano, J.I. (2005): Sistemas de información geográfica y evaluación multicriterio en la ordenación del territorio. Madrid, RA-MA.

GRAFCAN (2010-2011): Modelos digitales de terreno [MAPA]. 1:5.000. Santa Cruz de Tenerife.

Gutiérrez Puebla, J., y Gould, M. (2000): SIG: sistemas de información geográfica. Madrid, Síntesis. 
García Cruz, J.I.; Febles Ramírez, M.F.; Pestana Pérez, G. (2017): “Las Tecnologías de la Información Geográfica aplicadas a la sistematización de los sistemas agrarios. Bases para el estudio de su dimensión espacial y temporal. El caso del mapa de cultivos de canarias (España)", GeoFocus (Artículos), $n^{\circ}$ 19, p. 181-206. ISSN: 1578-5157 http://dx.doi.org/10.21138/GF.558

Hernández Hernández, J.F.; Simancas Cruz, M.R. y García Cruz, J.I. (2010): "La insularidad en la configuración de especificidades regionales: su impacto sobre los modelos de organización y ordenación territorial de los espacios insulares de Canarias", en Simancas Cruz, M.R. (coord.): El impacto de la crisis en la economía canaria. Claves para el futuro, vol 2. San Cristóbal de La Laguna, Real Sociedad Económica de Amigos del País de Tenerife, Cabildo Insular de Tenerife, pp. 725-785.

ISTAC (2008): "Estadísticas del territorio". http://www.gobiernodecanarias.org/istac/jaxiistac/menu.do?uripub=urn:uuid:fbc0bdc8-cacb-43b8-a5cb-a93f745dcff6 [fecha de consulta: 22 de junio de 2017].

Martín Martín, V.O. (2000): “Aproximación tipológica a los paisajes agrarios actuales de Canarias", Papeles de Geografía, 32, pp. 97-115.

Parra López, C.; de Haro Giménez, T.; Calatrava Requena, J. (2004): Análisis multifuncional de sistemas agrarios: aplicación del método del proceso analítico jerárquico al olivar de producción convencional, ecológica e integrada en Andalucía. Málaga, Edit. Unicaja.

Pestana Pérez, G.; Febles Ramírez, M. F. y de la Rosa Villar, B. [coord.] (2015): La agricultura Canaria a principios del siglo XXI. Análisis de los mapas de cultivos de Canarias. Madrid, Ministerio de Agricultura, Alimentación y Medio Ambiente.

Pro Ruiz, J. (1995): “Ocultación de la riqueza rústica en España (1870-1936): acerca de la fiabilidad de las estadísticas sobre la propiedad y uso de la tierra", Revista de Historia Económica, 1, pp. 89114.

Simancas Cruz, M.R. (2007): Las áreas protegidas de Canarias. Cincuenta años de protección ambiental del territorio en espacios insulares. Santa Cruz de Tenerife, Edit. Idea.

Tapiador, F. J. (2006): Las Tecnologías de Información Geográfica: Criticando al crítico. Una respuesta a Horacio Capel. Ciudad, Biblio 3W. Revista Bibliográfica de Geografía y Ciencias Sociales. [Consulta: 19-12-2016]. Disponible en http://www.ub.edu/geocrit/b3w-646.htm

Tomlinson, R. (2007): Pensando en el SIG. Planificación del sistema de información geográfica dirigida a gerentes. Redlands, ESRI PRESS.

Vallejo Pousada, R. (2000): "Los amillaramientos como fuente estadística: una visión crítica desde la contribución territorial”, Historia Agraria, 20, pp. 95-122.

Vallejo Pousada, R. (2010): “La estadística territorial española desde 1845 a 1900 ¿por qué no se hizo un catastro en el siglo XIX?", TC: Catastro, 68, pp. 81-115.

Villalba Moreno, E. (1978): Estudio del cultivo del tomate en Tenerife y Gran Canaria. Santa Cruz de Tenerife. Santa Cruz de Tenerife, Gráficas Tenerife.

Zurita Espinosa, L. (2011): La gestión del conocimiento territorial. Madrid, RA-MA. 